hep-th/0105168

CTP-MIT-3131

PUPT-1986

NSF-ITP-01-34

\title{
Boundary CFT Construction of D-branes in Vacuum String Field Theory
}

\author{
Leonardo Rastelli $^{a}$, Ashoke Sen $^{b}$ and Barton Zwiebach ${ }^{c}$ \\ ${ }^{a}$ Department of Physics \\ Princeton University, Princeton, NJ 08540, USA \\ E-mail: rastelli@feynman.princeton.edu \\ ${ }^{b}$ Harish-Chandra Research Institute \\ Chhatnag Road, Jhusi, Allahabad 211019, INDIA \\ and \\ Institute for Theoretical Physics \\ University of California, Santa Barbara, CA 93106, USA \\ E-mail: asen@thwgs.cern.ch, sen@mri.ernet.in \\ ${ }^{c}$ Center for Theoretical Physics \\ Massachussetts Institute of Technology, \\ Cambridge, MA 02139, USA \\ E-mail: zwiebach@mitlns.mit.edu
}

\begin{abstract}
In previous papers we built (multiple) D-branes in flat space-time as classical solutions of the string field theory based on the tachyon vacuum. In this paper we construct classical solutions describing all D-branes in any fixed space-time background defined by a two dimensional CFT of central charge 26. A key role is played by the geometrical definition of the sliver state in general boundary CFT's. The correct values for ratios of D-brane tensions arise because the norm of the sliver solution is naturally related to the disk partition function of the appropriate boundary CFT. We also explore the possibility of reproducing the known spectrum of physical states on a D-brane as deformations of the sliver.
\end{abstract}




\section{Contents}

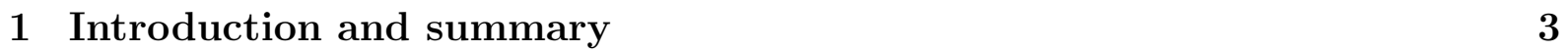

\begin{tabular}{|lll}
2 & Review of vacuum string field theory & 6
\end{tabular}

\begin{tabular}{|lll}
\hline 3 & The various pictures of the sliver & $\mathbf{8}$
\end{tabular}

3.1 Viewpoints on surface states . . . . . . . . . . . . . . . . . . . . . . . . . 8

3.2 The sliver surface state defined . . . . . . . . . . . . . . . . . . . . . 11

3.3 Using SL(2,R)-to resolve singular coordinates . . . . . . . . . . . . . . . . 14

3.4 Star multiplication of surface states . . . . . . . . . . . . . . . . . . . . . . 20

\begin{tabular}{|lll}
4 & General D-brane solutions & 23
\end{tabular}

4.1 Solution describing an arbitrary D-brane . . . . . . . . . . . . . . . . . . . 24

4.2 Multiple D-branes and coincident D-branes . . . . . . . . . . . . . . . . . . 26

4.3 Finite deformations of the sliver . . . . . . . . . . . . . . . . . . . . . . . . 28

4.4 Computation of the tension . . . . . . . . . . . . . . . . . . . . . 30

4.5 Small deformations of the sliven . . . . . . . . . . . . . . . . . . . . 32

4.6 Background independence and theory-space connections . . . . . . . . . . . 33

\begin{tabular}{|lll}
5 & Physical states around D-brane backgrounds & 36
\end{tabular}

$5.1 \quad$ Factorization ansatz for fluctuations . . . . . . . . . . . . . . . . . . . . . . 37

5.2 Factorization ansatz for gauge transformations . . . . . . . . . . . . . . . . 40

5.3 Deformed projectors and rules for physical states . . . . . . . . . . . . . . 41

\begin{tabular}{lll}
\hline 6 & Discussion & 43
\end{tabular} 


\section{Introduction and summary}

Much work has been done in understanding various conjectures about tachyon condensation on D-branes in bosonic string theory [1, 2, 3] using cubic open string field theory [4] (SFT). Although the results are very impressive, they ultimately rely on numerical study of the solutions of the equations of motion using the level truncation scheme $15,6,7,8,9,10,11,12,13,14,15,16,17,18,19,20,21,22,23,24.1$ In a series of three papers [29, 30, 31] we attempted an analytic approach to the issues of tachyon condensation by proposing a candidate string field theory action which describes string field theory expanded around the tachyon vacuum. As opposed to the conventional cubic SFT where the kinetic operator is the BRST operator $Q_{B}$, here the kinetic operator $\mathcal{Q}$ is non-dynamical and is built solely out of worldsheet ghost fields. In this class of actions the absence of physical open string states around the vacuum is manifest. Gauge invariance holds, and therefore basic consistency requirements are expected to be satisfied. Furthermore we showed that this theory contains classical solutions representing D-p-branes for all $p \leq 25$, with correct ratios of tensions, thereby providing a non-trivial check on the correctness of our proposal. The key ansatz that made this analysis possible was that the string field solution representing a D-brane factorizes into a ghost part $\Psi_{g}$ and a matter part $\Psi_{m}$, with $\Psi_{g}$ the same for all D-branes, and $\Psi_{m}$ different for different D-branes. The $\Psi_{m}$ representing the D25 brane is a particular surface state of the corresponding boundary conformal field theory (BCFT) known as the sliver [11, 33, 30, 31].

In this paper we discuss the construction of D-brane solutions in this theory using conformal field theory techniques. This construction has certain conceptual and practical advantages. In the construction of ref. [30] we obtained an expression for the ratio of tensions of different D-branes in terms of ratios of determinants of infinite matrices. There was no analytic understanding, however, why this ratio gave the expected answer. In the approach taken here, the correct values of all ratios of tensions are obtained manifestly. Furthermore the analysis given in this paper can be carried out in the background of any bulk conformal field theory (CFT), for any D-brane described by an appropriate BCFT.5

We start with some fixed space-time background described by a particular bulk CFT, and choose once and for all a specific reference BCFT, denoted as $\mathrm{BCFT}_{0}$. We define our string field to be a state in the Hilbert space of $\mathrm{BCFT}_{0}$. In the analysis of refs. 29, 30, 31]

\footnotetext{
${ }^{1}$ For some early attempts at understanding the open string tachyon, see refs. 25]. For field theory models of tachyon condensation, see refs. [26, 27]. Study of tachyon condensation using renormalization group approach has been carried out in refs. 28].

${ }^{2} \mathrm{~A}$ subset of this class of actions was discussed previously in ref. [32].

${ }^{3}$ Throughout this paper a specific BCFT will refer to the boundary conformal field theory associated with a single D-brane. Multiple D-brane solutions will be obtained by first constructing solutions corresponding to the individual D-branes, and then taking appropriate superposition of the solutions.
} 
the D25-brane BCFT played the role of $\mathrm{BCFT}_{0}$. As in those works, we seek solutions of the form $\Psi_{g} \otimes \Psi_{m}$, where the ghost component $\Psi_{g}$ is universal, but the matter part $\Psi_{m}$ varies from one solution to another. Under this factorization hypothesis, the matter part of the string field satisfies a simple equation: it squares to itself under $*$-multiplication. One particular solution of this equation is the matter part of the sliver state of $\mathrm{BCFT}_{0}$. We identify this state as the solution representing the D-brane associated with $\mathrm{BCFT}_{0}$, generalizing the identification of the D25-brane solution as the sliver of the corresponding BCFT [30, 31].

In the conformal field theory description [11, the sliver is regarded as a surface state by using the standard procedure for associating a BCFT state to every Riemann surface with a boundary, with one puncture at the boundary and a local coordinate at the puncture. The sliver is described as the surface state associated with a specific once-punctured disk. This description of the sliver is universal in the sense that the state takes exactly the same form for any BCFT when written in terms of the Virasoro operators of the BCFT. It is therefore natural to expect that, just as in the case of $\mathrm{BCFT}_{0}$, the sliver of an arbitrary BCFT describes the D-brane associated with that BCFT. In order to regard different D-branes as different solutions in the SFT, however, we need to express the slivers associated with these different BCFT's as states in the Hilbert space of $\mathrm{BCFT}_{0}$. We find an explicit algorithm for doing this. The solution constructed this way correctly reproduces the tension of the D-brane associated with the specific BCFT (up to an overall normalization constant which is the same for all D-branes). This is a nontrivial result that follows because the tension, in vacuum SFT, is given by the BPZ inner product of the solution with itself, and we show that this inner product is simply related to the disk partition function in the specific BCFT. The identification of the disk partition function with the D-brane tension is a well-known result [34, 35, 36, 37, 38] that has played a crucial role in the study of tachyon condensation in boundary string field theory [39, 40, 41, 42, 43, 44, 45, 46, 47, 48.

The paper is organized as follows. In section 2 we give a brief summary of the results of refs. [29, 30]. In section 3 we give a detailed review of the construction of the sliver as a surface state associated with a once-punctured disk with a local coordinate at the puncture. We describe it in various coordinate systems which are useful in our analysis. We also explain why it is a well defined state, in that the inner product of the sliver with any BCFT state associated with a vertex operator is computable and finite. This is not a priori obvious, since the state arises in a limit where the local coordinate at the puncture becomes singular. Nevertheless, SL $(2, R)$ invariances of the state allow geometrical representations where the local coordinate is non-singular, and the resulting state is manifestly regular. It is significant that even the regular geometrical description of the 
sliver is unusual in that the open string midpoint, which usually is somewhere inside the disk, reaches the boundary of the disk. This fact gives an intuitive understanding of the left-right factorization of the sliver functional, a key result in the analysis of [31, 49]. Our discussion also addresses star multiplication of surface states, and explains why the sliver squares to itself under star multiplication.

In section 4 we use the universal description of the sliver to construct solutions of the SFT equations of motion describing different D-branes in some fixed space-time background. We take for the space-time background some fixed bulk CFT with central charge 26 , and choose a reference boundary conformal field theory $\mathrm{BCFT}_{0}$, in whose state space the string field takes value. The matter part of the sliver of $\mathrm{BCFT}_{0}$ is the matter part of a solution describing the D-brane corresponding to $\mathrm{BCFT}_{0}$. We show in section 4.1 that every other D-brane in this space-time background, described by some other boundary conformal field theory BCFT', can also be obtained as a solution in this SFT. We explicitly write down the classical solution describing this D-brane, and verify that it satisfies the equations of motion and correctly reproduces the tension of the D-brane. In section 4.2 we show that solutions describing D-branes associated with two different BCFT's *multiply to zero, and hence we can construct multiple D-brane solutions by superposing them. We also give a construction of identical coincident D-branes.

In section 4.3 we discuss the construction of classical solutions corresponding to a two dimensional field theory obtained by deforming $\mathrm{BCFT}_{0}$ by a relevant or marginal boundary operator. The results bear strong resemblance to those of boundary string field theory, except for one important difference. In boundary string field theory, the coefficient of a relevant deformation is driven to infinity (or more generally to the infrared fixed point) by the equations of motion. In contrast, here we get a solution of the equations of motion for arbitrary value of this coefficient. When we compute the tension of the corresponding solution in section 4.4, however, we recover the partition function on a disk of the deformed boundary conformal field theory (which we call $\mathrm{BCFT}^{\prime}$ ), with the coefficient of the perturbation driven to its infrared fixed point due to a conformal transformation involving infinite rescaling. Thus the conclusion is that different values of these coefficients describe the same D-brane solution - the one associated with $\mathrm{BCFT}^{\prime}$.t For exactly marginal deformations, of course, different values of the coefficient represent genuinely different solutions.

If $\mathrm{BCFT}^{\prime}$ and $\mathrm{BCFT}_{0}$ are related by an exactly marginal deformation, we can also study small deformations of the solution by taking the perturbing parameter to be small. This is done in section 4.5. We show in section 4.6 that these small deformations can be thought of as covariant derivatives of the sliver state with respect to a canonical theory-

\footnotetext{
${ }^{4}$ This had been earlier anticipated by Witten [50].
} 
space connection introduced in ref. [51]. We also discuss the background independence of vacuum string field theory in the language of connections over theory space.

In section 5 we address the question of the spectrum of physical states around the solution describing the D-brane associated with $\mathrm{BCFT}_{0}$. Since general excitations around this solution involve both matter and ghost oscillators, this problem cannot be studied completely without knowing the form of the kinetic operator $\mathcal{Q}$ and the ghost part $\Psi_{g}$ of the D-brane solution. We make an attempt to study this problem in section 5.1 by restricting ourselves to excitations of the factorized type with the same universal ghost part $\Psi_{g}$ but arbitrary fluctuations in the matter sector. We find that we do get solutions of the linearized equations of motion for every dimension-one primary in the matter part of $\mathrm{BCFT}_{0}$, as is expected of the open string spectrum on a D-brane. We also need to determine which of these solutions could be related by linearized gauge transformations. Again, without detailed knowledge of $\mathcal{Q}$, we proceed with a restricted class of gauge transformation parameters described in section 5.2 which preserve the factorized form of the linearized fluctuations. Section 5.3 describes some subtleties that arise in studying the normalization of states. We discuss a plausible resolution leading to some interesting conclusions. First, it is strongly suggested that the kinetic operator $\mathcal{Q}$ of vacuum SFT must annihilate the identity operator. Second, we find that any linearized solution to the equations of motion with finite BPZ norm is pure gauge. Physical states are then argued to be associated with states whose BPZ norm diverges logarithmically in the parameters defining finite versions of the sliver. Although these criteria lead us to identify sliver deformations by dimension-one primaries as physical states, it is not clear how dimension one nulls and non-primary operators are removed from the spectrum.

We conclude in section 6 with a discussion of some general aspects of the string field theory around the tachyon vacuum.

\section{Review of vacuum string field theory}

In this section we shall briefly describe the results of refs. [29, 30]. In these papers we proposed a form of the string field theory action around the open bosonic string tachyon vacuum and discussed classical solutions describing D-branes of various dimensions. In order to write concretely this theory we choose to use the state space $\mathcal{H}$ of the combined matter-ghost boundary conformal field theory (BCFT) describing the D25-brane. The string field $\Psi$ is a state of ghost number one in $\mathcal{H}$ and the string field action is given by:

$$
\mathcal{S}(\Psi) \equiv-\frac{1}{g_{0}^{2}}\left[\frac{1}{2}\langle\Psi, \mathcal{Q} \Psi\rangle+\frac{1}{3}\langle\Psi, \Psi * \Psi\rangle\right]
$$


where $g_{0}$ is the open string coupling constant, $\mathcal{Q}$ is an operator made purely of ghost fields, $\langle$,$\rangle denotes the BPZ inner product, and *$ denotes the usual $*$-product of the string fields [4]. $\mathcal{Q}$ satisfies the requirements:

$$
\begin{aligned}
& \mathcal{Q}^{2}=0, \\
& \mathcal{Q}(A * B)=(\mathcal{Q} A) * B+(-1)^{A} A *(\mathcal{Q} B), \\
& \langle\mathcal{Q} A, B\rangle=-(-)^{A}\langle A, \mathcal{Q} B\rangle .
\end{aligned}
$$

The action (2.1) is then invariant under the gauge transformation:

$$
\delta \Psi=\mathcal{Q} \Lambda+\Psi * \Lambda-\Lambda * \Psi
$$

for any ghost number zero state $\Lambda$ in $\mathcal{H}$. Ref. 29] contains candidate operators $\mathcal{Q}$ satisfying these constraints; for our analysis we shall not need to make a specific choice of $\mathcal{Q}$. The equations of motion are

$$
\mathcal{Q} \Psi+\Psi * \Psi=0
$$

In ref. 30] we made the ansatz that all D-p-brane solutions in this theory have the factorized form:

$$
\Psi=\Psi_{g} \otimes \Psi_{m},
$$

where $\Psi_{g}$ denotes a state obtained by acting with the ghost oscillators on the $\mathrm{SL}(2, \mathrm{R})$ invariant vacuum of the ghost BCFT, and $\Psi_{m}$ is a state obtained by acting with matter operators on the $\mathrm{SL}(2, \mathrm{R})$ invariant vacuum of the matter BCFT. Let us denote by $*^{g}$ and $*^{m}$ the star product in the ghost and matter sector respectively. Eq.(2.4) then factorizes as

$$
\mathcal{Q} \Psi_{g}=-\Psi_{g} *^{g} \Psi_{g}
$$

and

$$
\Psi_{m}=\Psi_{m} *^{m} \Psi_{m}
$$

We further assumed that the ghost part $\Psi_{g}$ is universal for all D-p-brane solutions. Under this assumption the ratio of energies associated with two different D-brane solutions, with matter parts $\Psi_{m}^{\prime}$ and $\Psi_{m}$ respectively, is given by:

$$
\frac{\left\langle\Psi_{m}^{\prime} \mid \Psi_{m}^{\prime}\right\rangle_{m}}{\left\langle\Psi_{m} \mid \Psi_{m}\right\rangle_{m}},
$$

with $\langle\cdot \mid \cdot\rangle_{m}$ denoting BPZ inner product in the matter BCFT. Thus the ghost part drops out of this calculation.

In ref. 30] we constructed analytically the matter part of the solution for different D$p$-branes, and verified numerically that we get the correct ratio of tensions of D-p-branes 
using eq.(2.8). The matter part of the D-25-brane solution was given by the sliver state $|\Xi\rangle$ which will play an important role in the analysis of this paper. The construction of $|\Xi\rangle$ will be reviewed in section 3 .

In this paper, we shall be using a more general setup. Instead of restricting ourselves to D-branes in flat space-time, we shall consider a general space-time background described by some arbitrary bulk CFT. The role of the D25-brane, in whose Hilbert space the string field takes value, is played by some fixed D-brane in this background associated with a specific BCFT. We shall call this $\mathrm{BCFT}_{0}$. The matter part of $|\Xi\rangle$, described as a surface state in $\mathrm{BCFT}_{0}$, then describes the $\mathrm{D}$-brane associated with $\mathrm{BCFT}_{0}$.

\section{The various pictures of the sliver}

In this section we will examine the sliver state $|\Xi\rangle$ from its geometrical definition. In this context we take the opportunity to describe in detail the various ways the sliver can be presented. We emphasize how, despite its origin as a surface state with a singular coordinate, it is a well-defined state satisfying $\Xi * \Xi=\Xi$. Although we are interested in the matter part of the sliver, we shall work in most part with the full sliver including the ghost sector, so that there is no central charge contribution to the various conformal transformations and gluing operations. Having established that $\Xi * \Xi=\Xi$, we can then use the factorization property of the $*$-product to conclude that $\Xi_{m} *^{m} \Xi_{m}=\Xi_{m}$ with suitable normalization of $\Xi_{m}$ (which could be infinite, but is universal in the sense that it does not depend on the specific choice of matter BCFT).

\subsection{Viewpoints on surface states}

The sliver is a ghost number zero state that has a universal definition. It is a surface state, which means that for any given BCFT it can be defined as the bra $\langle\Xi|$ associated to a particular Riemann surface $\Sigma$. The surface in question is a disk $D$ with one puncture $P$ at the boundary. Moreover, there is a local coordinate at this puncture. The local coordinate at the puncture for the case of the sliver is obtained by a limiting procedure, to be reviewed and elaborated below.

We shall begin with a general discussion of surface states associated with a disk with one puncture. A local coordinate at a puncture is obtained from an analytic map $m$ taking a canonical half-disk $H_{U}$ defined as

$$
H_{U}:\{|\xi| \leq 1, \Im(\xi) \geq 0\},
$$

into $D$, where $\xi=0$ maps to the puncture $P$, and the image of the real segment $\{|\xi| \leq$ $1, \Im(\xi)=0$ \} lies on the boundary of $D$. The coordinate $\xi$ of the half disk is called the 


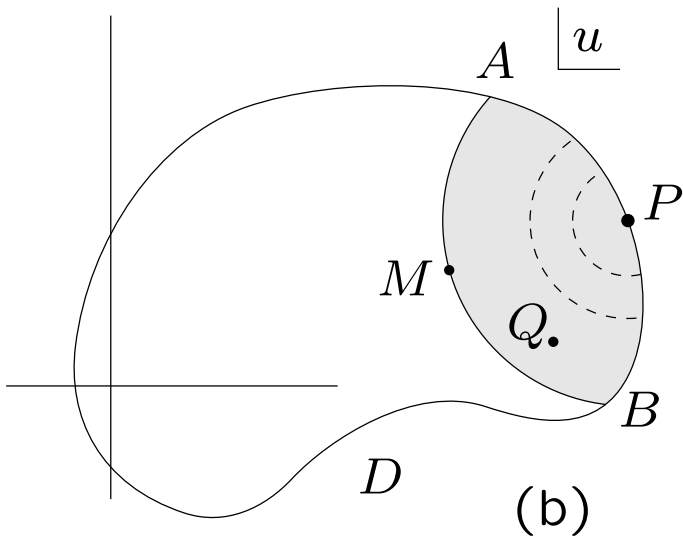

(b)

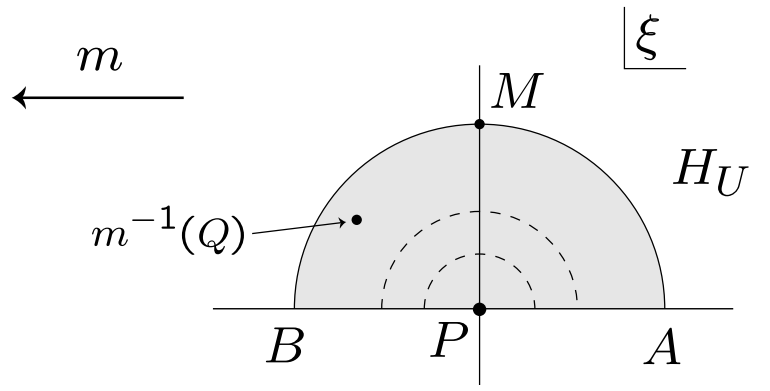

(a)

Figure 1: A punctured disk $D$ with a local coordinate around the puncture $P$. The coordinate is defined through a map $m$ from a canonical half disk $H_{U}$ to the disk. The arcs $A M$ and $M B$ in $D$ represent the left half and the right half of the open string respectively.

local coordinate. For any point $Q \in D$ in the image of the map, $\xi\left(m^{-1}(Q)\right)$ is the local coordinate of the point. Using any global coordinate $u$ on the disk $D$, the map $m$ can be described by some analytic function $s$ :

$$
u=s(\xi), \quad u(P)=s(0) .
$$

Figure 1 shows a disk $D$ with a local coordinate around the puncture $P$. The image under $m$ of the semicircle $|\xi|=1$, corresponding to the curve $A M B$ in Fig. 11 is usually referred to as the open string. The image of point $M$, corresponding to $\xi=i$, is called the string midpoint. The image of the arc $A M$ is called the left-half (as seen from the interior of $D$ ) of the open string, and similarly the image of $M B$ is called the right-half of the open string. If we denote by $\sigma=\frac{1}{i} \ln \xi$ the coordinate along the string, then the left-half of the string corresponds to the region $0 \leq \sigma<\frac{\pi}{2}$ and the right-half of the string corresponds to the region $\frac{\pi}{2}<\sigma \leq \pi$. The image of the half-disk $H_{U}$ in the $u$-plane, shown by the shaded region in Fig.1, will be called the local coordinate patch.

Given this geometrical data, and a BCFT with state space $\mathcal{H}$, the state $\langle\Sigma| \in \mathcal{H}^{*}$ associated to the surface $\Sigma$ is defined as follows. For any local operator $\phi(\xi)$, with associated state $|\phi\rangle=\lim _{\xi \rightarrow 0} \phi(\xi)|0\rangle$ we set

$$
\langle\Sigma \mid \phi\rangle=\langle s \circ \phi(0)\rangle_{D}
$$

where \langle\rangle$_{D}$ corresponds to correlation function on $D$ and $s \circ \phi(0)$ denotes the conformal transform of the operator by the map $s(\xi)$, i.e. the operator $\phi(\xi=0)$ expressed using the 
appropriate conformal map in terms of $\phi(s(0))$. For a primary of dimension $h, s \circ \phi(0)=$ $\phi(s(0))\left(s^{\prime}(0)\right)^{h}$. The right hand side of eq.(3.3) can be interpreted as the one point function on $D$ of the local operator $\phi$ inserted at $P$ using the local coordinate $\xi$ defined there. We also call, with a small abuse of notation, $|\Sigma\rangle \in \mathcal{H}$ a surface state; this is simply the BPZ conjugate of $\langle\Sigma|$. While computations of correlation functions involving states in $\mathcal{H}$ requires that the map $s$ be defined only locally around the puncture $P$, more general constructions, such as the gluing of surfaces, an essential tool in the operator formulation of CFT, requires that the full map of the half disk $H_{U}$ into the disk $D$ be well defined.

At an intuitive level $\langle\Sigma|$ can be given the following functional integral representation. Consider the path integral over the basic elementary fields of the two dimensional conformal field theory, - collectively denoted as $\varphi,-$ on the disk $D$ minus the local coordinate patch, with some fixed boundary condition $\varphi=\varphi_{0}(\sigma)$ on the boundary $A M B$ of the local coordinate patch, and the open string boundary condition corresponding to the BCFT under study on the rest of the boundary of this region. The parameter $\sigma$ is the coordinate labeling the open string along $A M B$, defined through $\xi=e^{i \sigma}$. The result of this path integral will a functional of the boundary value $\varphi_{0}(\sigma)$. We identify this as the wave-functional of the state $\langle\Sigma|$. (For describing the wave-functional of $|\Sigma\rangle$ we need to make a $\sigma \rightarrow(\pi-\sigma)$ transformation.) On the other hand the wave-functional of the state $|\phi\rangle$ can be obtained by performing the path integral over $\varphi$ on the unit half-disk in the $\xi$ coordinate system, with the boundary condition $\varphi=\varphi_{0}(\sigma)$ on the semicircle, open string boundary condition corresponding to the BCFT on real axis, and a vertex operator $\phi(0)$ inserted at the origin. We can now compute $\langle\Sigma \mid \phi\rangle$ for any state $|\phi\rangle$ in $\mathcal{H}$ by multiplying the two wave-functionals and integrating over the argument $\varphi_{0}(\sigma)$. The net result is a path integration over $\varphi$ on the full disk $D$, with the boundary condition corresponding to $\mathrm{BCFT}$ over the full boundary and a vertex operator $\phi$ inserted at the puncture $P$ using the $\xi$ coordinate system. This is precisely eq.(3.3).

For future use, we shall now describe three canonical ways of defining the surface state $\langle\Sigma|$, using three presentations of the disk $D$. In the first one, we present $D$ as the unit disk $D_{0}:|w| \leq 1$ in a $w$-plane. The puncture will be located at $w=1$, and the local coordinate described as

$$
w=\check{f}(\xi), \quad w(P)=\check{f}(0)=1 .
$$

This is shown in Figure 2(b). In this presentation we have that eq.(3.3) takes the form

$$
\langle\Sigma \mid \phi\rangle=\langle\check{f} \circ \phi(0)\rangle_{D_{0}}
$$

In the second presentation of $D$ we map it to the upper half plane (UHP) with global coordinate $z$, and locate the puncture at $z=0$. We will denote this upper half-plane as 
$D_{H}$. More concretely, we define $z$ through the relation

$$
w=h(z) \equiv \frac{1+i z}{1-i z},
$$

which maps the UHP, labeled by $z$, to the unit disk $D_{0}$. We then have that the local coordinate around $P$ in this presentation takes the form:

$$
z=h^{-1}(w)=h^{-1}(\check{f}(\xi)) \equiv \tilde{f}(\xi)
$$

In this presentation eq. (3.3) can be rewritten as

$$
\langle\Sigma \mid \phi\rangle=\langle\tilde{f} \circ \phi(0)\rangle_{D_{H}},
$$

where \langle\rangle$_{D_{H}}$ denotes correlation function on the upper half plane. The disk $D_{H}$ with its coordinate is shown in figure $2(\mathrm{c})$.

Finally, we introduce the third presentation, where the disk $D$ is mapped into a disk $\widehat{D}$ having the special property that the local coordinate patch, i.e. the image of the half disk $H_{U}$ in $\widehat{D}$, is particularly simple. With global coordinate $\widehat{w}$, the image of $H_{U}$ appears as a vertical half-disk of unit radius, with the curved part of $H_{U}$ mapped to the imaginary axis and the diameter of $H_{U}$ mapped to the unit semi-circle to the right of the imaginary axis (see Fig.2(d)). This is achieved by taking, for $\xi \in H_{U}$,

$$
\widehat{w}=h(\xi)=\frac{1+i \xi}{1-i \xi} .
$$

In this presentation the rest of $\widehat{D}$ may take a complicated form. We can now rewrite eq. (3.3) as

$$
\langle\Sigma \mid \phi\rangle=\langle h \circ \phi(0)\rangle_{\widehat{D}},
$$

where \langle\rangle$_{\widehat{D}}$ denotes the correlation function on the disk $\widehat{D}$ with appropriate open string boundary condition at the boundary of $\widehat{D}$. In this description the information about the state is encoded in the shape of the disk $\widehat{D}$.

\subsection{The sliver surface state defined}

We can now define the sliver state following the route originally taken through a limit of certain 'wedge states' 11]. We shall give this description in all three pictures by explicitly specifying the maps $\breve{f}(\xi), \widetilde{f}(\xi)$ and the disk $\widehat{D}$. In doing so we will refer to Fig. 3 , 国, and 5. We begin by giving the description on the unit disk $D_{0}$. We define for any positive real number $n>0$

$$
w_{n}=\check{f}_{n}(\xi) \equiv(h(\xi))^{2 / n}=\left(\frac{1+i \xi}{1-i \xi}\right)^{2 / n},
$$



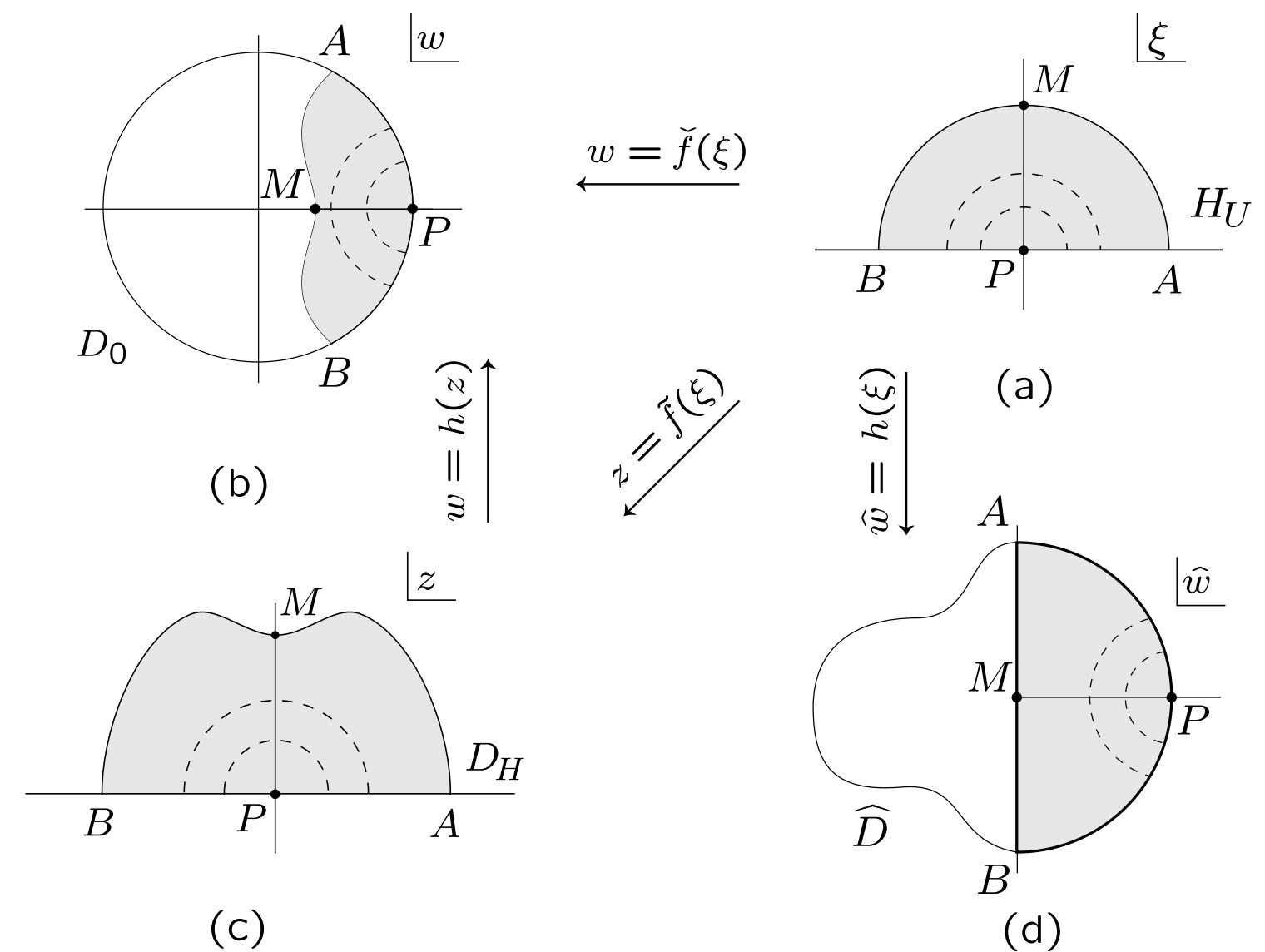

(c)

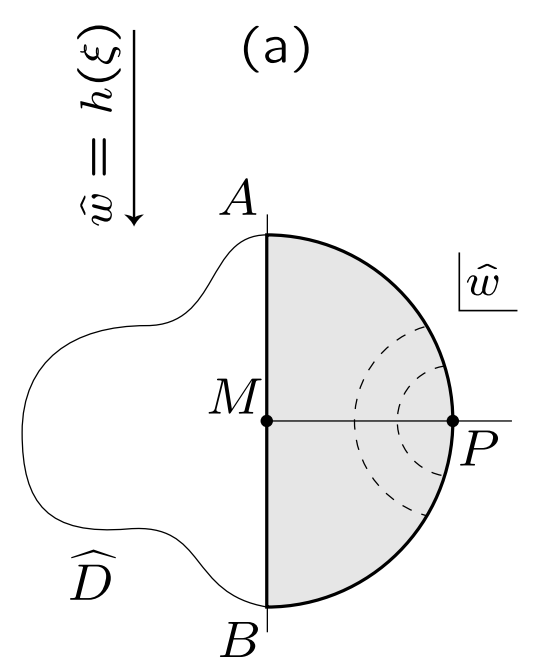

(d)

Figure 2: Three canonical presentations of the disk $D$. (b) Disk presented as the unit disk $D_{0}$ with global coordinate $w$. (c) Disk presented as the upper half plane $D_{H}$ with global coordinate $z$. (d) Disk presented as $\widehat{D}$ with global coordinate $\widehat{w}$. Here the image of $H_{U}$ is also a half-disk.

which for later purposes we also write as

$$
w_{n}=\exp \left(i \frac{4}{n} \tan ^{-1}(\xi)\right) .
$$

As already pointed out before, the map $h(\xi)$ takes the canonical half disk into a similar presentation, with the puncture now on the curved side of a half-disk (Figure $3-b)$. Moreover the string midpoint $M$ at $\xi=i$ is mapped to $h(i)=0$. The map $w_{n}=(h(\xi))^{2 / n}$ makes the image of the canonical half-disk into a wedge with the angle at $w_{n}=0$ equal to $2 \pi / n$. Figure (3-c) shows the disk $D_{0}$ in the $w_{n}$-plane with the puncture and the local coordinate. For any fixed $n$ we call the $\langle n|$ the resulting surface state. Thus we have

$$
\langle n \mid \phi\rangle \equiv\left\langle\check{f}_{n} \circ \phi(0)\right\rangle_{D_{0}} \quad \forall \phi .
$$



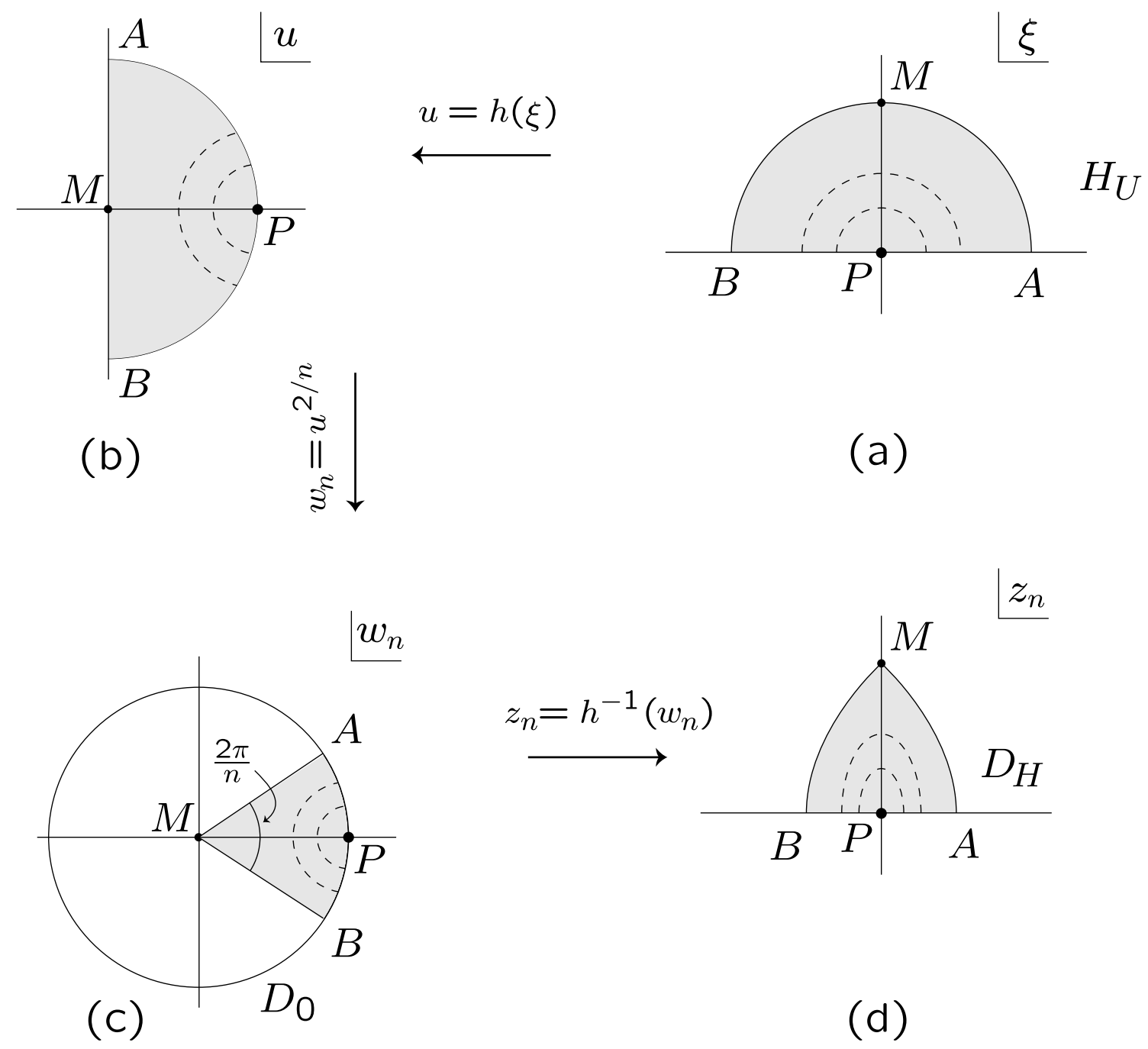

Figure 3: The definition of wedge states for arbitrary $n$. (a) The canonical half disk $H_{U}$. (b) The map of $H_{U}$ into a vertical half-disk. (c) The inclusion of the vertical half-disk of (b) into the disk $D_{0}$ with global coordinate $w$. (d) The map of $D_{0}$ into the upper half plane $D_{H}$.

The state obtained when $n=1$ is the identity state (see Figure 4 a). For this state the local coordinate patch in the $w_{n}$ plane covers the full unit disk $D_{0}$ with a cut on the negative real axis. The left-half and the right-half of the string coincide along this cut. The state $n=2$ is the vacuum state. In this case the image of $H_{U}$ covers the right half of the full unit disk $D_{0}$ in the $w_{n}$ plane. In the $n \rightarrow \infty$ limit, the image of $H_{U}$ in the $w_{n}$ coordinate is a 'thin sliver' of the disk $D_{0}$ (Figure [-b). It was seen in [11] that the 
limit $n \rightarrow \infty$ of $\langle n|$ gives rise to a well-defined state. In the next subsection we shall give a detailed explanation for this result. This surface state $\langle\Xi|$, called the sliver, has the property that the left-half and the right-half of the string are as far as they can be on the unit disk.

Next we describe the state $|n\rangle$ using the coordinate $z$ on the upper half plane (see Fig.3-d). We have

$$
z_{n}=h^{-1}\left(w_{n}\right)=i \frac{1-w_{n}}{1+w_{n}}=\tan \left(-\frac{i}{2} \ln w_{n}\right) .
$$

The composition of (3.12) and (3.14) gives [1]

$$
z_{n}=\tan \left(\frac{2}{n} \tan ^{-1}(\xi)\right) \equiv \tilde{f}_{n}(\xi)
$$

and we have

$$
\langle n \mid \phi\rangle=\left\langle\tilde{f}_{n} \circ \phi(0)\right\rangle_{D_{H}} .
$$

Finally we introduce the coordinate $\widehat{w}$ for the presentation of the surface state $|n\rangle$ using a disk $\widehat{D}$. Using eqs. (3.9) and (3.11) we see that

$$
\widehat{w}_{n}=\left(w_{n}\right)^{n / 2} \text {. }
$$

This is simply the map inverse to that taking Fig. B-b to Fig. B-c, but extended for all of $D_{0}$. Under this map the unit disk $D_{0}$ in the $w_{n}$-coordinates is mapped to a cone in the $\widehat{w}_{n}$ coordinate, subtending an angle $n \pi$ at the origin $\widehat{w}_{n}=0$. We shall denote this cone by $\widehat{D}_{n}$. Thus we have

$$
\langle n \mid \phi\rangle=\langle h \circ \phi(0)\rangle_{\widehat{D}_{n}} .
$$

In the $n \rightarrow \infty$ limit $\widehat{D}_{n}$ can be viewed as an infinite helix, as shown in Fig.5.

\subsection{Using $\mathrm{SL}(2, \mathrm{R})$-to resolve singular coordinates}

All three descriptions of the sliver, using eqs.(3.13), (3.16) and (3.18), are apparently singular. (3.13) and (3.16) are singular since the corresponding maps $\check{f}_{n}(\xi)$ and $\tilde{f}_{n}(\xi)$, defined in eqs. 3.11) and (3.15) are singular in the $n \rightarrow \infty$ limit. On the other hand, (3.18) is singular since the region $\widehat{D}_{n}$ becomes a cone with infinite excess angle at the origin in the $n \rightarrow \infty$ limit. We shall now show that these are only apparent singularities, and that for any Fock space state $|\phi\rangle,\langle\Xi \mid \phi\rangle$ is a perfectly finite number.

The main ingredient of the proof will be the $\mathrm{SL}(2, \mathrm{R})$ invariance of the correlation functions in the upper half plane. Given any $\operatorname{SL}(2, \mathrm{R})$ map $R(z)$, we have the relation:

$$
\left\langle\prod_{i} \mathcal{O}_{i}\left(x_{i}\right)\right\rangle_{D_{H}}=\left\langle\prod_{i} R \circ \mathcal{O}_{i}\left(x_{i}\right)\right\rangle_{D_{H}}
$$




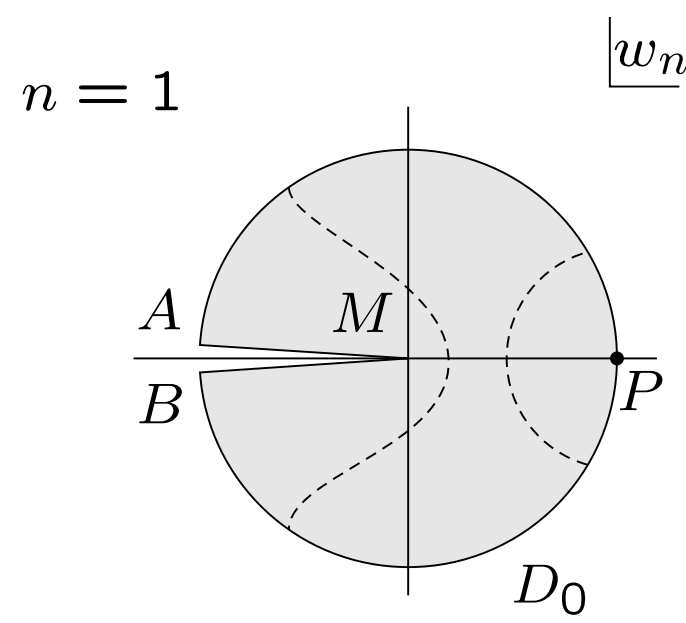

Identity $\langle\mathcal{I}|$

(a)

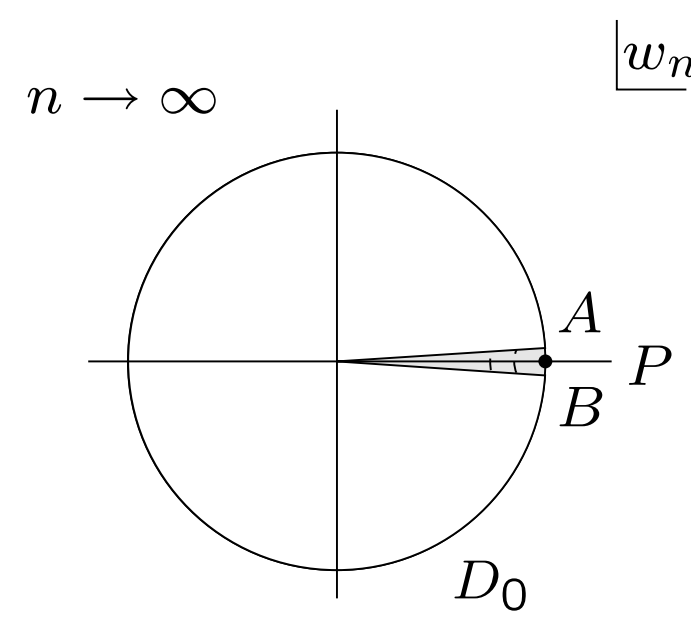

Sliver $\langle\underline{\underline{\Xi}}|$

(b)

Figure 4: (a) The surface state corresponding to the identity string field $\langle\mathcal{I}|$. Here the image of $H_{U}$ covers the full disk, except for a cut in the negative real axis. (b) The surfaces state corresponding to the sliver $\langle\Xi|$. Here the image of $H_{U}$ covers an infinitesimally thin sliver around the positive real axis.

for any set of operators $\mathcal{O}_{i}$ and with $D_{H}$ denoting the upper half plane. Thus we can rewrite equation (3.16) as:

$$
\langle n \mid \phi\rangle=\left\langle R_{n} \circ \tilde{f}_{n} \circ \phi(0)\right\rangle_{D_{H}},
$$

for any set of $\operatorname{SL}(2, \mathrm{R})$ maps $R_{n}$. Thus if we can find a sequence of maps $R_{n}$ such that $R_{n} \circ \widetilde{f}_{n}$ approaches a finite limit $f$ as $n \rightarrow \infty$ with $f(\xi)$ non-singular at the origin, then we can define the sliver $\langle\Xi|$ through the relations:

$$
\langle\Xi \mid \phi\rangle=\langle f \circ \phi(0)\rangle_{D_{H}} .
$$

In this case we choose:

$$
R_{n}(z)=\frac{n}{2} z
$$

so that

$$
\begin{aligned}
f(\xi) & =\lim _{n \rightarrow \infty} R_{n} \circ \tilde{f}_{n}(\xi) \\
& =\lim _{n \rightarrow \infty} \frac{n}{2} \tan \left(\frac{2}{n} \tan ^{-1}(\xi)\right)=\tan ^{-1} \xi
\end{aligned}
$$



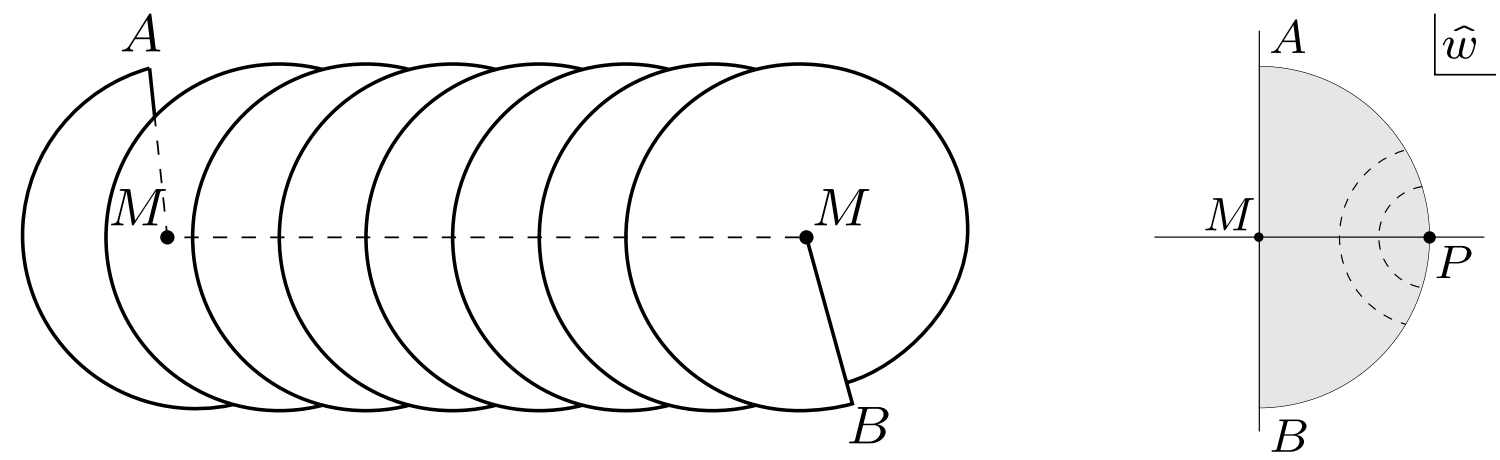

Figure 5: Using $\widehat{w}$ global coordinates the sliver appears as a cone with infinite excess anglenamely, an infinite helix. The segments $A M$ and $B M$ represent the left-half and the right-half of the string. The local coordinate patch, represented by the shaded half disk shown to the right, must be glued in to form the complete surface.

Since this map is non-singular at $\xi=0$, this provides a finite answer for $\langle\Xi \mid \phi\rangle$ for any Fock space state $|\phi\rangle$, thereby providing a non-singular description of the sliver. We shall denote by

$$
z^{\prime}=f(\xi)
$$

the new coordinate on $D_{H}$. This picture of the sliver state is shown in Figure 6(a,b). The local coordinate patch has become the full strip bounded by the lines $\Re\left(z^{\prime}\right)= \pm \pi / 4$. These two semiinfinite lines are the left- and right- halves of the string. They meet at the midpoint $M$ which has been sent to $z^{\prime}=i \infty$. This is actually the only reminder that the sliver state is a limit of a sequence of fully regular states. While the coordinate at the puncture can be taken to be regular at this limit, and thus the state $\langle\Xi|$ is welldefined, in this limit the map of $H_{U}$ into the disk fails to be regular at one point. The string midpoint has been sent to the boundary of the disk. While this does not affect any computation involving Fock space states, this fact is significant in that it shows that there is no fully regular geometric presentation of the sliver. This might cause some worry since, as mentioned before, for gluing purposes the image of the $\operatorname{arc}\{|\xi|=1,0<\arg (\xi)<\pi\}$ should be inside the disk. We shall see, however, that gluing operations involving the sliver can be made well-defined despite this singularity.

This description of the sliver can be obtained by considering another sequence of maps. For this we introduce a new coordinate system

$$
\widehat{z}_{n}=\frac{1}{2 i} \ln \widehat{w}_{n} .
$$




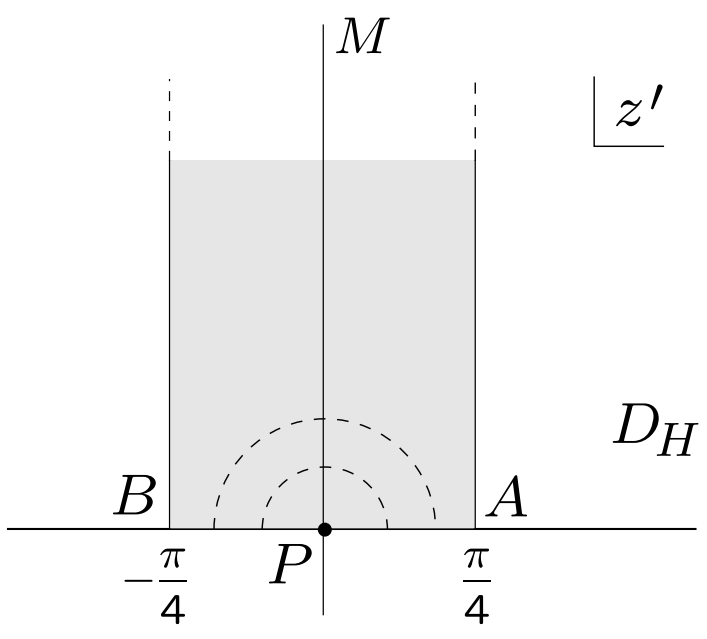

(b)

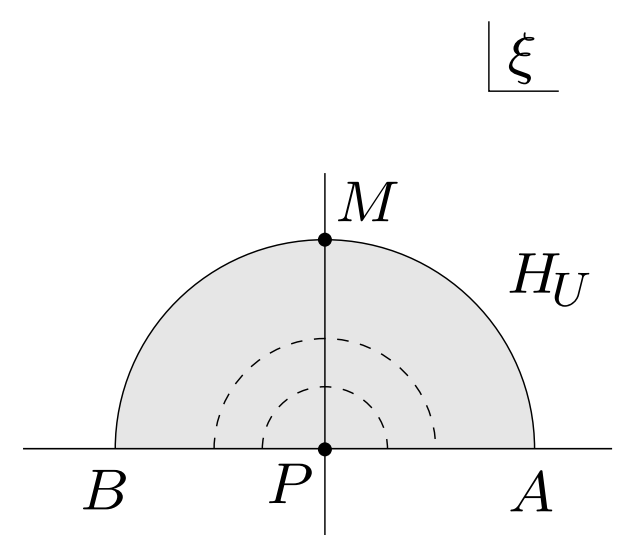

(a)

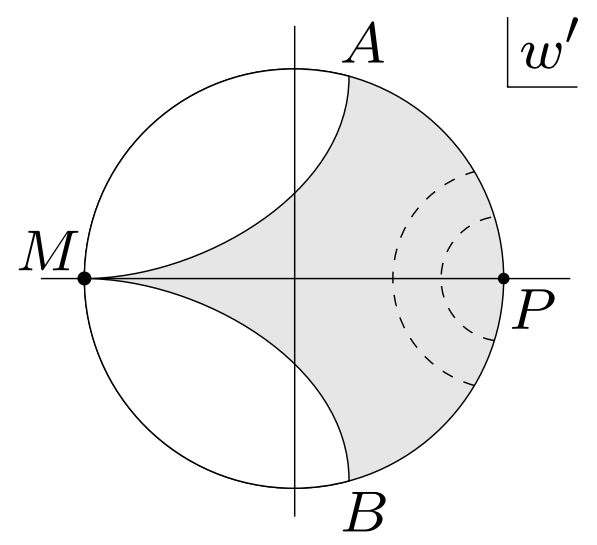

(c)

Figure 6: The regular presentations of the sliver surface state with non-singular coordinates at the puncture. In (b) the disk $D$ is the full upper half plane, and the image of the local coordinate disk $H_{U}$ is the vertical strip whose boundaries are the left-half and right-half of the string. The string midpoint is at infinity. (c) Mapping $D_{H}$ back to a unit disk with $w^{\prime}=h\left(z^{\prime}\right)$ we find a regular presentation on the disk.

The cone $\widehat{D}_{n}$ in the $\widehat{w}_{n}$ coordinate system maps to a semiinfinite cylinder $\widehat{C}_{n}$ in the $\widehat{z}_{n}$ coordinate system with $\widehat{z}_{n}$ spanning the range:

$$
-\frac{\pi}{4} \leq \Re\left(\widehat{z}_{n}\right) \leq\left(\frac{n}{2}-\frac{1}{4}\right) \pi, \quad \Im\left(\widehat{z}_{n}\right) \geq 0, \quad \widehat{z}_{n} \simeq \widehat{z}_{n}+n \frac{\pi}{2} .
$$


The local coordinate patch is the region:

$$
-\pi / 4 \leq \Re\left(\widehat{z}_{n}\right) \leq \pi / 4, \quad \Im\left(\widehat{z}_{n}\right) \geq 0 .
$$

This has been shown in Fig. 7. The relationship between $\widehat{z}_{n}$ and the local coordinate $\xi$ follows from eqs.(3.9) and (3.25):

$$
\widehat{z}_{n}=\tan ^{-1} \xi \equiv f(\xi) .
$$

Thus we have

$$
\langle n \mid \phi\rangle=\langle f \circ \phi(0)\rangle_{\widehat{C}_{n}} \quad \forall|\phi\rangle \in \mathcal{H} .
$$

Note now that using the periodicity along the $\Re\left(\widehat{z}_{n}\right)$ direction we could take the range of $\Re\left(\widehat{z}_{n}\right)$ to be $-n \pi / 4 \leq \Re\left(\widehat{z}_{n}\right) \leq n \pi / 4$. In this case as $n \rightarrow \infty, \widehat{C}_{n}$ approaches the full UHP and the coordinate $\widehat{z}_{n}$ approaches the coordinate $z^{\prime}$ introduced earlier.

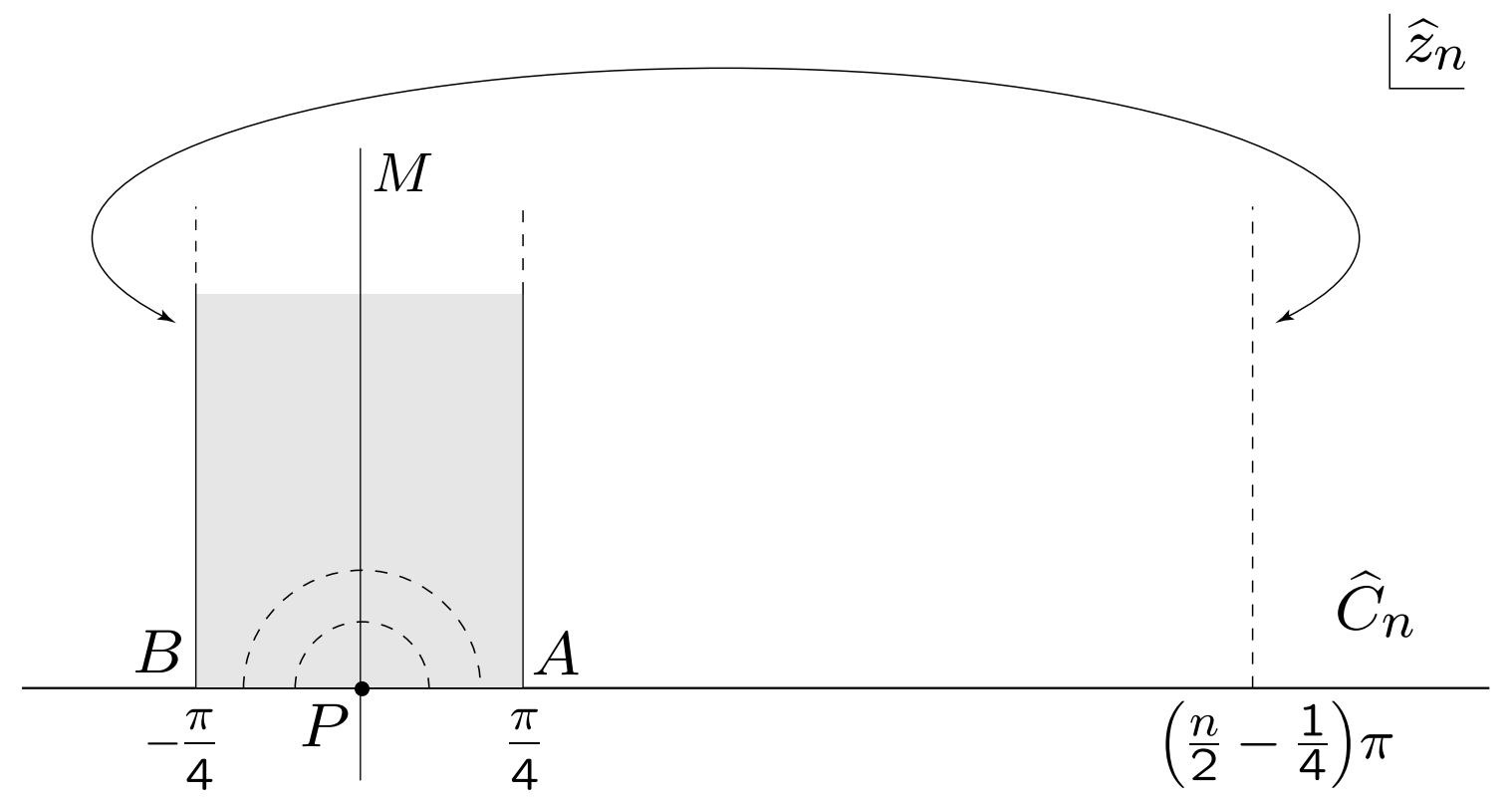

Figure 7: The finite $n$ approximation to the sliver presented in the $\widehat{z}_{n}$ coordinate.

Having obtained a regular description of the sliver we can map it back to the unit disk by writing $w^{\prime}=h\left(z^{\prime}\right)$, with $h$ defined in (3.6). This gives us Figure 6-c; the regular presentation in the disk. The only remnant of the singular origin is the fact that the open string midpoint reaches the boundary of the disk and the open string develops a cusp at the midpoint. As a result of this, at least intuitively, the surface representing the 
sliver appears to be cut into two disjoint pieces by the local coordinate patch. One could therefore expect the wavefunctional of the sliver to factorize into two wavefunctionals, one corresponding to the data on the left-half of the string, and the other to the data on the right-half of the string. It was seen in refs. [31, 49] that this intuitive expectation is indeed realized.

We would like to emphasize that the use of $\mathrm{SL}(2, \mathrm{R})$ invariance to resolve singular coordinates is most effective for once punctured disks. For the case of three punctured disks, the three (distinguishable) punctures can always be fixed at three points and there are no $\mathrm{SL}(2, \mathrm{R})$ invariances left. If the local coordinate at one puncture is singular, nothing can be done about it. This is not the case for once punctured disks, because after fixing the position of the puncture there are powerful conformal isometries left over. f For example, after fixing the puncture at $z=0$, the maps $\tilde{z}=a z /(c z+d)$ do change the looks of the local coordinate as shown in Figure 8. Even though the local coordinate patch in the $z$ or $\tilde{z}$ upper half planes look different, and even though the functions $\tilde{z}=\tilde{g}(\xi)$ and $z=g(\xi)$ are different, as Riemann surfaces with local coordinates they are indeed identical. For the case of two punctured disks, with punctures fixed at $z=0$ and $z=1$, for example, the maps $\tilde{z}=a z /(z+(a-1))$ preserve the punctures. Near $z=0$ the map looks like $\tilde{z}=a z /(a-1)$ and near $z=1$ it looks like $\tilde{z}=1+(z-1)(a-1) / a$. Since the scaling factors are inverses of each other, if only one coordinate is singular it cannot be resolved. Only particular cases when the coordinates are singular in a related way could be resolved.

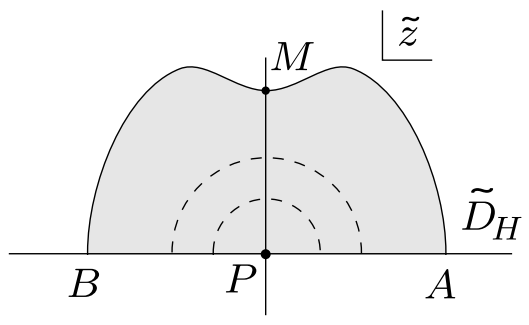

(c)

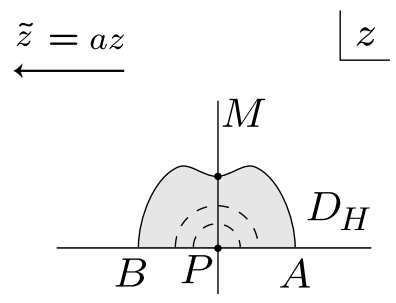

(b)

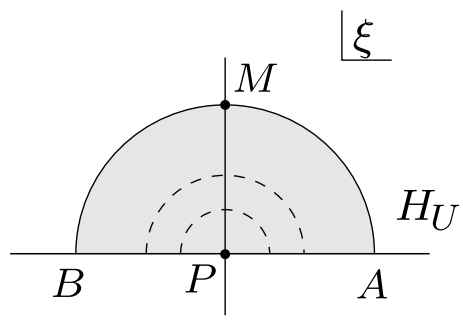

(a)

Figure 8: The power of conformal isometries in resolving singular coordinates on once-punctured disks is illustrated by showing two versions of a disk $\left(D_{H}\right.$ and $\left.\tilde{D}_{H}\right)$ that, while looking different, represent the same Riemann surface. Using an $a \rightarrow \infty$ limit one could resolve a vanishingly small image of $H_{U}$ in $D_{H}$.

5 This of course does not mean that any singularity of the local coordinate patch can be removed by such transformations, but there is a reasonable degree of freedom. 


\subsection{Star multiplication of surface states}

The star multiplication of two surface states is easiest to describe by representing them in the $\widehat{w}$ coordinate system. Let us assume that we have two such surface states, and associated with them are the regions $\widehat{D}$ and $\widehat{D}^{\prime}$, which describe the images of the disk in the corresponding $\widehat{w}$ coordinate system. Each of these disks contain as a subspace the region $h\left(H_{U}\right)$, - the local coordinate patch. We shall denote by $\mathcal{R}$ and $\mathcal{R}^{\prime}$ the complement of $h\left(H_{U}\right)$ in $\widehat{D}$ and $\widehat{D}^{\prime}$ respectively. By an abuse of notation we shall denote the surface states associated with the disks $\widehat{D}$ and $\widehat{D}^{\prime}$ by $|\mathcal{R}\rangle$ and $\left|\mathcal{R}^{\prime}\right\rangle$ respectively. This is represented diagrammatically in Figure 9, where in parts (a) and (b) we show two surface states $|\mathcal{R}\rangle$ and $\left|\mathcal{R}^{\prime}\right\rangle$ built in this way. The regions $h\left(H_{U}\right)$ have been shown shaded in this figure. In fact, once we have made it clear that the local coordinate is presented in the specific fashion chosen here, we could simply represent pictorially the surfaces as only the regions $\mathcal{R}$, namely the image of the full disk minus the local coordinate patch.f

If the surface states are presented this way, the star multiplication $|\mathcal{R}\rangle *\left|\mathcal{R}^{\prime}\right\rangle$ is readily performed. One removes the local coordinate patches from the disks $\widehat{D}$ and $\widehat{D}^{\prime}$, and glues the right half (as viewed from the region of $\widehat{D}$ outside the local coordinate patch) $M B$ of the $\mathcal{R}$ open string to the left half $A^{\prime} M^{\prime}$ of the $\mathcal{R}^{\prime}$ open string. The result is the composite region $\mathcal{R} \cdot \mathcal{R}^{\prime}$ shown in the figure. This region represents the star product of the states, namely

$$
|\mathcal{R}\rangle *\left|\mathcal{R}^{\prime}\right\rangle=\left|\mathcal{R} \cdot \mathcal{R}^{\prime}\right\rangle
$$

In this region, the string is $A M B^{\prime}$, with $A M$ the left half, and $M B^{\prime}$ the right half. The local coordinate patch shown to the right, must be glued in to produce the full picture of the surface state.

We now discuss the multiplication of wedge states $|n\rangle$. As we have already discussed, in the $\widehat{w}$ coordinate system relevant for the state $\langle n|$, the disk becomes a cone subtending an angle $\pi n$ at the origin. If we remove the local coordinate patch the left over region becomes a sector of angle $\pi(n-1)$. If we denote by $\left|\mathcal{R}_{\alpha}\right\rangle$ a sector state arising from a sector of angle $\alpha$, we have the identification of sector states with wedge states:

$$
|n\rangle=\left|\mathcal{R}_{\pi(n-1)}\right\rangle \text {. }
$$

Moreover, it is clear from our discussion above that star multiplication of sector states simply gives a sector whose angle is the sum of those in the product:

$$
\left|\mathcal{R}_{\alpha}\right\rangle *\left|\mathcal{R}_{\beta}\right\rangle=\left|\mathcal{R}_{\alpha+\beta}\right\rangle
$$

\footnotetext{
${ }^{6}$ Leaving off the local coordinate, of course, carries a small risk of confusion, especially because the local coordinate patch could be presented in other canonical ways, for example, as semi-infinite strips.
} 


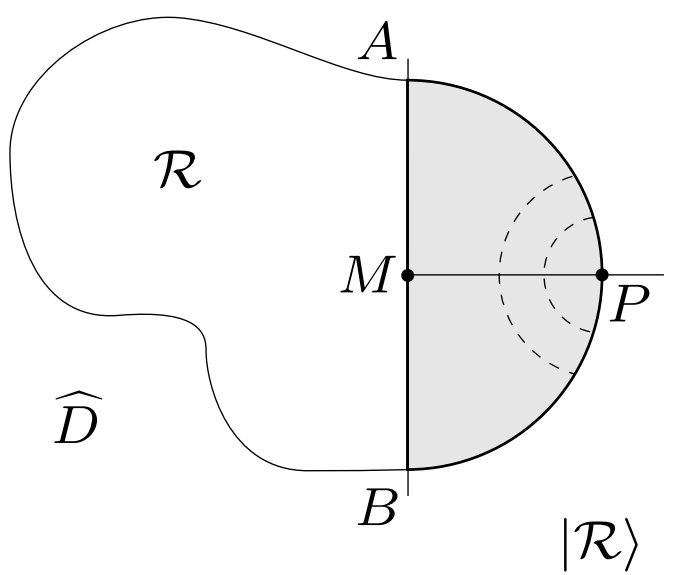

(a)

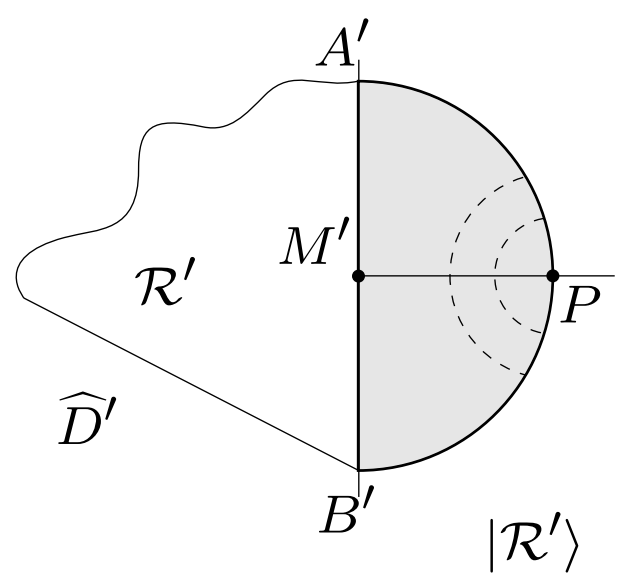

(b)

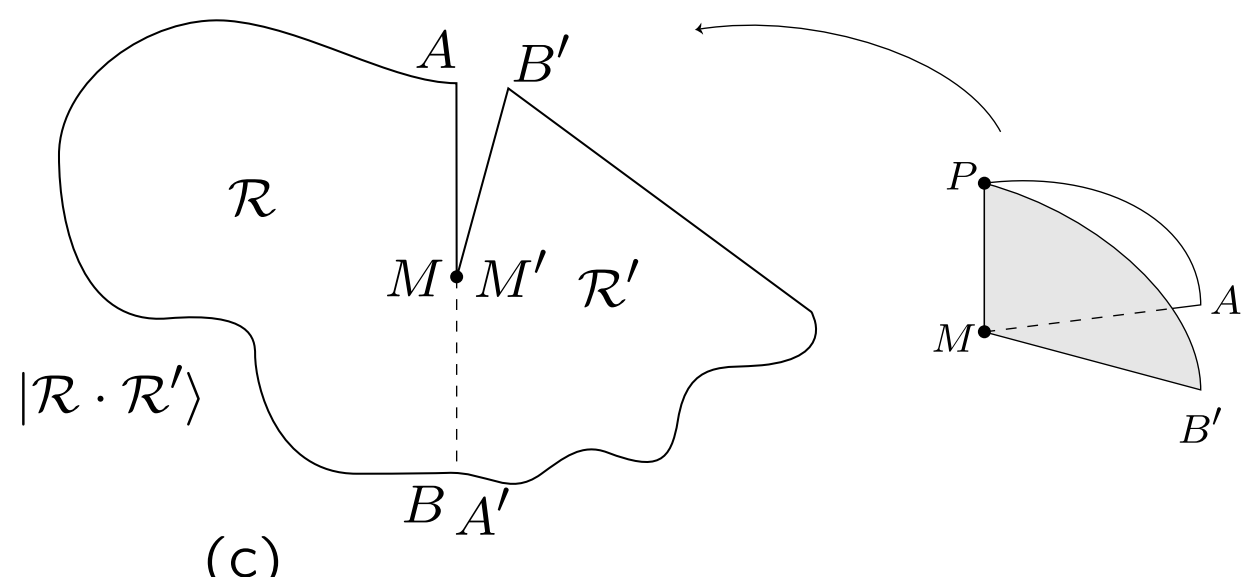

(c)

Figure 9: Star multiplication of surface states is simple if the states are presented with $\widehat{w}$ coordinates, in which case the local coordinate appears as a (shaded) half-disk in the disk $\widehat{D}$. Here are shown two surface states; $|\mathcal{R}\rangle$ in (a), and $\left|\mathcal{R}^{\prime}\right\rangle$ in (b). The star product is shown in (c) and is obtained gluing the regions $\mathcal{R}$ and $\mathcal{R}^{\prime}$ as indicated, and attaching the half disk (shown to the right) representing the local coordinate and the puncture.

This is illustrated in Figure 10. Indeed the multiplication rule (3.32) is consistent with $|n\rangle *|m\rangle=|n+m-1\rangle$, which is the familiar multiplication rule for wedge states. The

${ }^{7}$ We hope the reader will not be confused by the dual use of the term surface state. In the wedge states, the focus is on the local coordinate which looks like a wedge inside a unit disk, in the sector states, the focus is on the complement of the local coordinates in the disk when the local coordinate patch is 


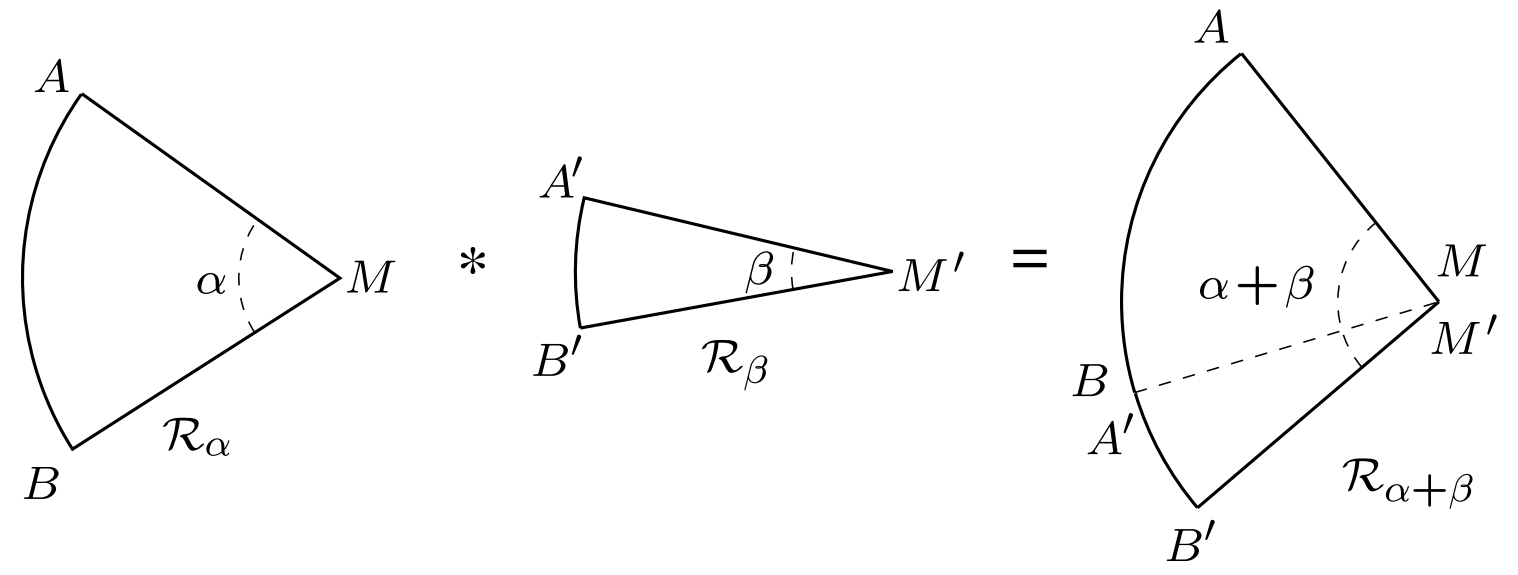

Figure 10: The star multiplication of a sector state with angle $\alpha$ to a sector state with angle $\beta$ gives a sector state with angle $\alpha+\beta$. Sector states are just another presentation of wedge states.

sliver arises from $n \rightarrow \infty$ and therefore corresponds to a sector state with infinite angle! This state was shown schematically in Figure 5. Indeed, it should be noted that among sector states only two can multiply to themselves, according to (3.32). One is the zeroangle sector state $\left|\mathcal{R}_{0}\right\rangle$ which is the identity $|\mathcal{I}\rangle$ (or the wedge state $|n=1\rangle$ ). The other is the sliver, which can be viewed as the zero angle wedge state, or as the infinite angle sector state. Indeed, from (3.32) we have $\left|\mathcal{R}_{\infty}\right\rangle *\left|\mathcal{R}_{\infty}\right\rangle=\left|\mathcal{R}_{\infty}\right\rangle$.

For later use it will be useful to work out the precise relationship between the different coordinate systems appearing in the description of the product state $\left|\mathcal{R}_{\alpha+\beta}\right\rangle$ and the states $\left|\mathcal{R}_{\alpha}\right\rangle$ and $\left|\mathcal{R}_{\beta}\right\rangle$. Again this is simple in the $\widehat{w}$ coordinate system. For this let us take

$$
\alpha=\pi(m-1), \quad \beta=\pi(n-1), \quad \alpha+\beta=\pi(m+n-2) .
$$

If we denote by $\widehat{w}_{m}, \widehat{w}_{n}$ and $\widehat{w}_{m+n-1}$ the $\widehat{w}$ coordinates associated with the sector states $\left|\mathcal{R}_{\alpha}\right\rangle,\left|\mathcal{R}_{\beta}\right\rangle$ and $\left|\mathcal{R}_{\alpha+\beta}\right\rangle$ respectively, we have

$$
\widehat{w}_{m+n-1}=\left\{\begin{array}{lll}
\widehat{w}_{m} & \text { in } & \mathcal{R}_{\alpha} \\
e^{i \alpha} \widehat{w}_{n} & \text { in } & \mathcal{R}_{\beta}
\end{array}\right.
$$

In the $\widehat{z}_{n}$ coordinate system introduced in eq.(3.25) the gluing relations (3.34) take a very presented as a half-disk. This complement looks like a sector. 
simple form:

$$
\widehat{z}_{m+n-1}= \begin{cases}\widehat{z}_{m} & \text { for } \quad \frac{\pi}{4} \leq \Re\left(\widehat{z}_{m}\right) \leq\left(\frac{m}{2}-\frac{1}{4}\right) \pi \\ \widehat{z}_{n}+\frac{1}{2}(m-1) \pi & \text { for } \quad \frac{\pi}{4} \leq \Re\left(\widehat{z}_{n}\right) \leq\left(\frac{n}{2}-\frac{1}{4}\right) \pi\end{cases}
$$

\section{General D-brane solutions}

In this section we shall give deformations of the sliver to generate new solutions of the equations of motion representing D-branes described by general BCFT's. The general idea is simple. We denote by $\mathrm{BCFT}_{0}$ the reference $\mathrm{BCFT}$ in whose Hilbert space the string field takes value. The sliver of $\mathrm{BCFT}_{0}$, whose matter part represents the D-brane solution associated with $\mathrm{BCFT}_{0}$, is the surface state described in the previous section, with the specific boundary condition corresponding to $\mathrm{BCFT}_{0}$ on the boundary of the surface. To get a solution representing the single D-brane of some other boundary conformal field theory $\mathrm{BCFT}^{\prime}$ we must represent the sliver of $\mathrm{BCFT}^{\prime}$ on the state space of $\mathrm{BCFT}_{0}$, as we now explain. The construction assumes that $\mathrm{BCFT}_{0}$ and $\mathrm{BCFT}^{\prime}$ have the same bulk conformal field theory, but of course, differ in their boundary interactions.

Usually the sliver $\Xi^{\prime}$ of $\mathrm{BCFT}^{\prime}$ will be described in the same manner as discussed in the last section, with all the correlation functions now being calculated in BCFT'. This, however, would express $\Xi^{\prime}$ as a state in the Hilbert space $\mathcal{H}^{\prime}$ of $\mathrm{BCFT}^{\prime}$, since in eq.(3.21), for example, $\phi$ will now represent a vertex operator of $\mathrm{BCFT}^{\prime}$. In order to express $\Xi^{\prime}$ in the Hilbert space of $\mathrm{BCFT}_{0}$, we adopt the following procedure. As discussed in section 3.2, at an intuitive level, the wave-functional for $\left\langle\Xi^{\prime}\right|$ is a functional of $\varphi_{0}(\sigma)$ with $\sigma=\frac{1}{i} \ln \xi$ labeling the coordinate along the string, obtained by functional integration over the two dimensional fields $\varphi$ on the full disk (UHP in this case) minus the local coordinate patch, with boundary condition $\varphi=\varphi_{0}(\sigma)$ on the boundaries $\Re\left(z^{\prime}\right)= \pm \pi / 4$, and the boundary condition appropriate to $\mathrm{BCFT}^{\prime}$ on the rest of the boundary of this region ( $z^{\prime}$ real, $\left.\left|\Re\left(z^{\prime}\right)\right| \geq \pi / 4\right)$. On the other hand given a state $|\phi\rangle$ in the Hilbert space $\mathcal{H}$ of $\mathrm{BCFT}_{0}$, we represent the wave-functional of $|\phi\rangle$ as a functional of $\varphi_{0}(\sigma)$, obtained by performing path integral over $\varphi$ in the inside of the local coordinate patch with boundary condition appropriate for $B C F T_{0}$ on the real axis, the vertex operator $\phi$ of $\mathrm{BCFT}_{0}$ inserted at the origin in the local coordinate system $\xi$, and the boundary condition $\varphi=\varphi_{0}(\sigma)$ on the semi-circle. Finally in order to calculate $\left\langle\Xi^{\prime} \mid \phi\right\rangle$ we take the product of the wavefunctional of $\left\langle\Xi^{\prime}\right|$ and the wave-functional of $|\phi\rangle$ and integrate over $\varphi_{0}(\sigma)$. The result will be a functional integral over the fields $\varphi$ on the full UHP, with boundary condition appropriate to $\mathrm{BCFT}^{\prime}$ in the range $\left|\Re\left(z^{\prime}\right)\right| \geq \pi / 4$, boundary condition corresponding to $\mathrm{BCFT}_{0}$ for $\left|\Re\left(z^{\prime}\right)\right| \leq \pi / 4$ and the vertex operator $\phi$ inserted at the origin in the local 
coordinate system. This can be expressed as

$$
\left\langle\Xi^{\prime} \mid \phi\right\rangle=\langle f \circ \phi(0)\rangle^{\prime \prime}
$$

where \langle\rangle$^{\prime \prime}$ denotes correlation function in a theory where we have BCFT' boundary condition for $\left|\Re\left(z^{\prime}\right)\right| \geq \pi / 4$ and $\mathrm{BCFT}_{0}$ boundary condition for $\left|\Re\left(z^{\prime}\right)\right| \leq \pi / 4$. $\phi$ is a vertex operator in $\mathrm{BCFT}_{0}$ and $f(\xi)=\tan ^{-1} \xi$ as usual.

In what follows, we shall show that after appropriate ultraviolet regularization, $\Xi^{\prime}$ defined this way squares to itself under $*$-multiplication, and also has the right tension for describing a D-brane associated to BCFT'. We will begin by considering this general case in the next subsection. Then we will discuss the situation where BCFT' is replaced by a general two dimensional field theory obtained from $\mathrm{BCFT}_{0}$ by some boundary perturbation.

\subsection{Solution describing an arbitrary D-brane}

We shall now describe the construction of the solution of the SFT equations of motion describing a D-brane corresponding to an arbitrary boundary conformal field theory $\mathrm{BCFT}^{\prime}$ with the same bulk CFT as BCFT $\mathrm{B}_{0}$. We start with the definition of $\Xi^{\prime}$ given in eq.(4.1). The effect of the change of the boundary condition beyond $\left|x^{\prime}\right| \geq \pi / 4$ can be represented by inserting boundary condition changing vertex operator $\sigma^{ \pm}$(discussed e.g. in [52, 53]) at $x^{\prime}= \pm \pi / 4$. In other words we can express (4.1) as

$$
\left\langle\Xi^{\prime} \mid \phi\right\rangle=\left\langle f \circ \phi(0) \sigma^{+}\left(\frac{\pi}{4}\right) \sigma^{-}\left(-\frac{\pi}{4}\right)\right\rangle .
$$

If we denote by $\mathrm{D}$ and $\mathrm{D}^{\prime}$ the $\mathrm{D}$-branes associated with $\mathrm{BCFT}_{0}$ and $\mathrm{BCFT}^{\prime}$ respectively, then $\sigma^{+}$denotes the vertex operator for the ground state of an open string whose left end (viewed from inside the UHP) is on the $\mathrm{D}^{\prime}$-brane and right end is on the D-brane, whereas $\sigma^{-}$denotes the vertex operator for the ground state of open string whose left end is on the D-brane and right end is on the $\mathrm{D}^{\prime}$-brane. In anticipation of short-distance divergences, we shall actually put $\sigma^{-}$and $\sigma^{+}$at $\left(-\frac{\pi}{4}-\epsilon\right)$ and $\left(\frac{\pi}{4}+\epsilon\right)$ respectively, where $\epsilon$ is a small positive number. We shall also use the description of the sliver as limit of finite $n$ wedge states in the $\widehat{z}_{n}$ coordinate introduced in eq.(3.25). Thus we have

$$
\left\langle\Xi^{\prime} \mid \phi\right\rangle=\lim _{n \rightarrow \infty}\left\langle f \circ \phi(0) \sigma^{+}\left(\frac{\pi}{4}+\epsilon\right) \sigma^{-}\left(\frac{n}{2} \pi-\frac{\pi}{4}-\epsilon\right)\right\rangle_{\widehat{C}_{n}} .
$$

We now calculate $\Xi^{\prime} * \Xi^{\prime}$. From the gluing relations (3.35) we get,

$$
\begin{aligned}
\left\langle\Xi^{\prime} * \Xi^{\prime} \mid \phi\right\rangle= & \lim _{m, n \rightarrow \infty}\left\langle f \circ \phi(0) \sigma^{+}\left(\frac{\pi}{4}+\epsilon\right) \sigma^{-}\left(\left(\frac{m}{2}-\frac{1}{4}\right) \pi-\epsilon\right)\right. \\
& \left.\sigma^{+}\left(\left(\frac{m}{2}-\frac{1}{4}\right) \pi+\epsilon\right) \sigma^{-}\left(\frac{1}{2}(m+n-1) \pi-\frac{\pi}{4}-\epsilon\right)\right\rangle_{\widehat{C}_{m+n-1}} .
\end{aligned}
$$


Thus the $\sigma^{ \pm}$at $\frac{1}{2}\left(m-\frac{1}{2}\right) \pi \pm \epsilon$ come close as $\epsilon \rightarrow 0$ and give rise to a divergent factor $(2 \epsilon)^{-2 h}$ where $h$ is the conformal weight of $\sigma^{ \pm}$. Hence we have $\Xi^{\prime} * \Xi^{\prime}=(2 \epsilon)^{-2 h} \Xi^{\prime}$. This requires us to redefine $\Xi^{\prime}$ by absorbing a factor of $(2 \epsilon)^{2 h}$, so that it squares to itself under *-multiplication

$$
\Xi_{R}^{\prime} \equiv(2 \epsilon)^{2 h} \Xi^{\prime} \quad \rightarrow \quad \Xi_{R}^{\prime} * \Xi_{R}^{\prime}=\Xi_{R}^{\prime} .
$$

We note, however, that even for finite $\epsilon$, the state $\Xi_{R}^{\prime}$ still squares to itself. Indeed, the product $\sigma^{+}\left(\frac{1}{2}\left(m-\frac{1}{2}\right) \pi+\epsilon\right) \sigma^{-}\left(\frac{1}{2}\left(m-\frac{1}{2}\right) \pi-\epsilon\right)$ can be expanded in an operator product expansion, and since these operators are moved to $\infty$ in the $m, n \rightarrow \infty$ limit, only the identity operator in this operator product expansion would contribute. The coefficient of the identity operator is given by $(2 \epsilon)^{-2 h}$ even for finite $\epsilon$.

Since $\mathrm{BCFT}_{0}$ and $\mathrm{BCFT}^{\prime}$ differ only in the matter sector, it is clear that $\left|\Xi_{R}^{\prime}\right\rangle$ has the factorized form

$$
\left|\Xi_{R}^{\prime}\right\rangle=\left|\Xi_{g}\right\rangle \otimes\left|\Xi_{R, m}^{\prime}\right\rangle,
$$

where $\Xi_{g}$ is a universal ghost factor. Normalizing $\Xi_{g}$ (which is independent of the choice of $\left.\mathrm{BCFT}^{\prime}\right)$ such that $\Xi_{g} *^{g} \Xi_{g}=\Xi_{g}$, we can ensure that

$$
\Xi_{R, m}^{\prime} *^{m} \Xi_{R, m}^{\prime}=\Xi_{R, m}^{\prime} .
$$

Thus we can now construct a new D-brane solution by taking the product $\left|\Psi_{g}\right\rangle \otimes\left|\Xi_{R, m}^{\prime}\right\rangle$, where $\left|\Psi_{g}\right\rangle$ is the same universal ghost state that appears in the construction of the D-brane solution corresponding to $\mathrm{BCFT}_{0}$.

We shall now calculate the tension associated with this new D-brane solution. For this we need to compute $\left\langle\Xi_{R, m}^{\prime} \mid \Xi_{R, m}^{\prime}\right\rangle_{m}$, where the subscript $m$ denotes matter. We have,

$$
\left\langle\Xi_{R, m}^{\prime} \mid \Xi_{R, m}^{\prime}\right\rangle_{m}=(2 \epsilon)^{4 h}\left\langle\Xi_{m}^{\prime} \mid \Xi_{m}^{\prime}\right\rangle_{m} .
$$

Calculation of $\left\langle\Xi_{m}^{\prime} \mid \Xi_{m}^{\prime}\right\rangle_{m}$ is again simple in the $\widehat{z}_{n}$ coordinate system. We first compute the $*$-product of the two states, and then in the final glued surface with coordinate $\widehat{z}_{m+n-1}$ we remove the local coordinate patch $-\pi / 4 \leq \Re\left(\widehat{z}_{m+n-1}\right) \leq \pi / 4$ and identify the lines $\Re\left(\widehat{z}_{m+n-1}\right)= \pm \pi / 4$. This produces the semi-infinite cylinder $\widetilde{C}_{m+n-2}$ defined by $\frac{\pi}{4} \leq \Re\left(z_{m+n-2}\right) \leq \frac{\pi}{4}+(m+n-2) \frac{\pi}{2}$ and $\Im\left(z_{m+n-2}\right) \geq 0$. We therefore find

$$
\begin{aligned}
\left\langle\Xi_{m}^{\prime} \mid \Xi_{m}^{\prime}\right\rangle_{m}= & \lim _{m, n \rightarrow \infty}\left\langle\sigma^{+}\left(\frac{\pi}{4}+\epsilon\right) \sigma^{-}\left(\left(\frac{m}{2}-\frac{1}{4}\right) \pi-\epsilon\right)\right. \\
& \left.\left.\sigma^{+}\left(\frac{m}{2}-\frac{1}{4}\right) \pi+\epsilon\right) \sigma^{-}\left(\frac{1}{2}(m+n-1) \pi-\frac{\pi}{4}-\epsilon\right)\right\rangle_{\widetilde{C}_{m+n-2}},
\end{aligned}
$$

where the correlation function is now being computed in the matter BCFT. We get a factor of $(2 \epsilon)^{-2 h}$ coming from $\sigma^{ \pm}$inserted at $\left(\frac{m}{2}-\frac{1}{4}\right) \pi \pm \epsilon$ as before, but there is another 
factor of $(2 \epsilon)^{-2 h}$ coming from the other $\sigma^{ \pm}$insertions that happen at points separated by a (minimal) distance $2 \epsilon$ on the cylinder $\widetilde{C}_{m+n-2}$. These exactly cancel the explicit factor of $(2 \epsilon)^{4 h}$ in (4.8). Since from the definition of $\sigma^{ \pm}$it is clear that in the $\epsilon \rightarrow 0$ limit we have BCFT' boundary condition on the full real $z_{m+n-2}$ axis, we find that $\left\langle\Xi_{R, m}^{\prime} \mid \Xi_{R, m}^{\prime}\right\rangle_{m}$ is given by the partition function of the deformed boundary CFT on the $\widetilde{C}_{m+n-2}$ cylinder

$$
\left\langle\Xi_{R, m}^{\prime} \mid \Xi_{R, m}^{\prime}\right\rangle_{m}=Z_{\widetilde{C}_{m+n-2}}\left(\mathrm{BCFT}^{\prime}\right) \sim Z_{D_{0}}\left(\mathrm{BCFT}^{\prime}\right)
$$

where in the last step we relate this partition function to the one on the standard unit disk. This is possible because of conformal invariance. Any constant multiplicative factor that might appear due to conformal anomaly depends only on the bulk central charge and is independent of the choice of $\mathrm{BCFT}^{\prime}$. This can at most give rise to a universal multiplicative factor. Since the partition function of $\mathrm{BCFT}^{\prime}$ on the unit disk is proportional to the tension of the corresponding D-brane [34, 35, 36, 37, 38], we see that the tension $\left\langle\Xi_{R, m}^{\prime} \mid \Xi_{R, m}^{\prime}\right\rangle_{m}$ computed from vacuum string field theory agrees with the known tension of the BCFT' D-brane, up to an overall constant factor independent of BCFT'.

Arguments similar to the one given for $\Xi^{\prime} * \Xi^{\prime}$ show that the result (4.10) holds even when $\epsilon$ is finite. In this case we have two pairs of $\sigma^{ \pm}$on the boundary, with the first pair being infinite distance away from the second pair. Thus we can expand each pair using operator product expansion and only the identity operator contributes, giving us back the partition function of $\mathrm{BCFT}^{\prime}$ on the disk. From this we see that we have a one parameter family of solutions, labeled by $\epsilon$, describing the same D-brane. We expect these solutions to be related by gauge transformations, since $\partial_{\epsilon} \Xi_{R, m}^{\prime}$ has finite norm (as can be easily verified) and hence is pure gauge according to the arguments to be given in section 5 .

\subsection{Multiple D-branes and coincident D-branes}

We first consider the construction of a configuration containing various D-branes associated to different BCFT's. To this end, we note that the star product $\Xi^{\prime} * \Xi$ of the $\mathrm{BCFT}^{\prime}$ solution and the $\mathrm{BCFT}_{0}$ solution vanishes. Indeed, using the same methods as in the previous subsection, the computation of $\Xi^{\prime} * \Xi$ leads to the cylinder $\widehat{C}_{m+n-1}$ with a $\sigma^{+}$ insertion at $\frac{\pi}{4}+\epsilon$ and a $\sigma^{-}$insertion at $\left(\frac{m}{2}-\frac{1}{4}\right)-\epsilon$. In the $m, n \rightarrow \infty$ limit, $\sigma^{-}$moves off to infinity and as a result the correlation function vanishes since $\sigma^{-}$has dimension larger than zero as long as $B C F T_{0}$ and $B C F T$ are different. Similar arguments show that $\Xi * \Xi^{\prime}$ and $\left\langle\Xi_{m} \mid \Xi_{m}^{\prime}\right\rangle_{m}$ also vanish. Thus the matter part of $\Xi+\Xi_{R}^{\prime}$ is a new solution describing the superposition of the D-branes corresponding to $\mathrm{BCFT}_{0}$ and $\mathrm{BCFT}^{\prime}$. Since no special assumptions were made about $\mathrm{BCFT}_{0}$ nor $\mathrm{BCFT}^{\prime}$, it follows that $\Xi^{\prime} * \Xi^{\prime \prime}=\Xi^{\prime \prime} * \Xi^{\prime}=0$ and $\left\langle\Xi_{m}^{\prime} \mid \Xi_{m}^{\prime \prime}\right\rangle_{m}=0$ for any two different $\mathrm{BCFT}^{\prime}$ and $\mathrm{BCFT}^{\prime \prime}$, and hence we can superpose any 
number of slivers to form a solution. This in particular also includes theories which differ from each other by a small marginal deformation. Special cases of this phenomenon, in the case of D-branes in flat space-time, have been discussed in ref. 49].

This procedure, however, is not suitable for superposing D-branes associated with the same BCFT, i.e. for parallel coincident D-branes. For example, if we take BCFT' to differ from $\mathrm{BCFT}_{0}$ by an exactly marginal deformation with deformation parameter $\lambda$, then in the $\lambda \rightarrow 0$ limit the operators $\sigma^{ \pm}$both approach the identity operator (having vanishing conformal weight), and although the argument for the vanishing of $\Xi * \Xi^{\prime}$ holds for any non-zero $\lambda$, it breaks down at $\lambda=0$.

In order to construct a superposition of identical D-branes, one can proceed in a different way. First consider getting coincident $\mathrm{BCFT}_{0}$ branes. To this end we introduce a modified $\mathrm{BCFT}_{0}$ sliver

$$
\left\langle\Xi_{\chi} \mid \phi\right\rangle=\lim _{n \rightarrow \infty}\left\langle f \circ \phi(0) \chi^{+}\left(\frac{\pi}{4}+\epsilon\right) \chi^{-}\left(\frac{n}{2} \pi-\frac{\pi}{4}-\epsilon\right)\right\rangle_{\widehat{C}_{n}} .
$$

Here $\chi^{ \pm}$are a conjugate pair 8 of operators of $\mathrm{BCFT}_{0}$, having a common dimension $h$ greater than zero, and representing some excited states of the open string with both ends having BCFT $T_{0}$ boundary condition. Thus, throughout the real line we have $\mathrm{BCFT}_{0}$ boundary conditions. We require that the coefficient of the identity in the OPE $\chi^{-}(x) \chi^{+}(y)$ is given by $|x-y|^{-2 h_{i}}$, and that this OPE does not contain any other operator of dimension $\leq 0$.

The clear parallel between eqn. (4.11) and eqn. (4.3), describing the BCFT' D-brane, implies that an analysis identical to the one carried out in the previous section will show that:

1. This new state $\Xi_{\chi}$ (after suitable renormalization as in eq.(4.5)) squares to itself under $*$-multiplication.

2. The BPZ norm of the matter part of $\Xi_{\chi}$ is proportional to the partition function of $\mathrm{BCFT}_{0}$ on the unit disk.

3. $\Xi_{\chi}$ has vanishing $*$-product with $\Xi$.

Thus the matter part of this state gives another representation of the D-brane associated with $\mathrm{BCFT}_{0}$, and we can construct a pair of D-branes associated with $\mathrm{BCFT}_{0}$ by superposing the matter parts of $\Xi$ and $\Xi_{\chi}$.

This construction can be easily generalized to describe multiple $\mathrm{BCFT}_{0} \mathrm{D}$-branes. We construct different representations of the same D-brane by using different vertex operators

${ }^{8}$ We need to choose $\chi^{ \pm}$to be conjugates of each other so that the string field is hermitian. 
$\chi^{(i) \pm}$ in $\mathrm{BCFT}_{0}$ satisfying the 'orthonormality condition' that the coefficient of the identity operator in the OPE of $\chi^{(i)-}(x) \chi^{(j)+}(y)$ is given by $\delta_{i j}|x-y|^{-2 h_{i}}$, and that this OPE does not contain any other operator of dimension $\leq 0$. The correponding solutions $\Xi_{\chi^{(i)}}$ all have vanishing $*$-product with each other, and hence can be superposed to represent multiple D-branes associated with $\mathrm{BCFT}_{0}$.

If instead we want to construct a superposition of identical BCFT' D-branes, we need to replace $\sigma^{ \pm}$in eq.4.3) by another pair of vertex operators $\tau^{ \pm}$which represent some excited states of open strings with one end satisfying $\mathrm{BCFT}_{0}$ boundary condition and the other end satisfying BCFT' boundary condition. The corresponding state $\Xi_{\tau}^{\prime}$ will give another representation of the D-brane associated with $\mathrm{BCFT}^{\prime}$, having vanishing *product with $\Xi^{\prime}$ as long as the operator product of $\sigma^{-}$with $\tau^{+}$does not contain the identity operator or any other operator of dimension $\leq 0$. Hence we can superpose these solutions to construct new solutions.

This procedure of adding vertex operators near $\pm \pi / 4$ to create new solutions representing the same D-brane is the BCFT version of the use of excited states of half-strings [31, 49] for the same purpose.

\subsection{Finite deformations of the sliver}

We shall now consider a class of solutions associated with the sliver for boundary field theories which are not necessarily conformal. Let us begin with the description of the sliver of $\mathrm{BCFT}_{0}$ given in the $\widehat{z}_{n}$ coordinate system defined in eq.(3.25). We have from eq. (3.29):

$$
\langle\Xi \mid \phi\rangle=\lim _{n \rightarrow \infty}\langle f \circ \phi(0)\rangle_{\widehat{C}_{n}} \quad \forall|\phi\rangle \in \mathcal{H}
$$

with $f(\xi)=\tan ^{-1} \xi$, and $\langle\cdot\rangle$ denotes correlation function calculated in $\mathrm{BCFT}_{0}$. Now suppose $V$ is a local vertex operator in the matter sector of $\mathrm{BCFT}_{0}$. We define a new state $\left\langle\Xi^{V, \lambda}\right|$ through the relation: $]^{\prime}$

$$
\left\langle\Xi^{V, \lambda} \mid \phi\right\rangle=\lim _{n \rightarrow \infty}\left\langle\exp \left(-\lambda \int_{\frac{\pi}{4}}^{\left(\frac{n}{2}-\frac{1}{4}\right) \pi} V\left(x_{n}\right) d x_{n}\right) f \circ \phi(0)\right\rangle_{\widehat{C}_{n}} \quad \forall|\phi\rangle \in \mathcal{H},
$$

where $x_{n}=\Re\left(\widehat{z}_{n}\right), \lambda$ is a constant, and the integration is done over the real $\widehat{z}_{n}$ axis excluding the part that is inside the local coordinate patch. The description of this state can be made more transparent by taking the full range of $\Re\left(\widehat{z}_{n}\right)$ to be $\left[-\frac{n}{4} \pi, \frac{n}{4} \pi\right]$ with the local coordinate patch in the region $-\frac{\pi}{4} \leq \Re\left(\widehat{z}_{n}\right) \leq \frac{\pi}{4}$, and then identifying $\widehat{z}_{n}$ as the coordinate $z^{\prime}$ on the upper half plane in the $n \rightarrow \infty$ limit. This gives

$$
\left\langle\Xi^{V, \lambda} \mid \phi\right\rangle=\left\langle\exp \left(-\lambda \int_{\left|x^{\prime}\right|>\frac{\pi}{4}} V\left(x^{\prime}\right) d x^{\prime}\right) f \circ \phi(0)\right\rangle_{D_{H}},
$$

\footnotetext{
${ }^{9} \mathrm{~A}$ construction that is similar in spirit but uses a different geometry was suggested in ref. [48].
} 
where $x^{\prime}=\Re\left(z^{\prime}\right)$. This expression should be treated as a correlation function in a theory where on part of the boundary we have the usual boundary action corresponding to $\mathrm{BCFT}_{0}$, and on part of the boundary we have a modified boundary action obtained by adding the integral of $V$ to the original action. In defining this we need to use suitable operator ordering, and regularization and renormalization prescriptions to remove the short distance singularities (see e.g. ref.[54] for a discussion of this). Typically this requires $V$ to be of dimension $\leq 1$. In special cases, as discussed in 41, we may also be able to include operators of higher dimensions, but as we shall see later these do not generate new solutions. We shall not explicitly take into account the effects of ultra-violet divergences in our analysis, and hence the results of this and the next subsection will be somewhat formal. The analysis, however, can be made concrete in specific examples, e.g. of the kind discussed in refs. 41]. If we regard the effect of $\lambda$ deformation to be a change in the boundary condition, then (4.14) represents a correlation function with $\mathrm{BCFT}_{0}$ boundary condition in the range $-\frac{\pi}{4} \leq x \leq \frac{\pi}{4}$ and the modified boundary condition outside this range.

We shall now show that $\left|\Xi^{V, \lambda}\right\rangle$ satisfies the relation:

$$
\Xi^{V, \lambda} * \Xi^{V, \lambda}=\Xi^{V, \lambda} .
$$

To compute the $*$-product in the left hand side of the above equation, we use the $\widehat{z}_{n}$ coordinate system, keeping $n$ finite and taking the $n \rightarrow \infty$ limit at the end. The advantage of using the $\widehat{z}_{n}$ coordinate system is in the simplicity of the gluing relations (3.35); since the coordinate $\widehat{z}_{m+n-1}$ of the surface after gluing is related to the coordinates $\widehat{z}_{m}$ and $\widehat{z}_{n}$ of the original surfaces by simple shift, there is no conformal transformation of the factors $\int V\left(x_{n}\right) d x_{n}$ except for the appropriate change of range. Thus the result of computing $\Xi^{V, \lambda} * \Xi^{V, \lambda}$ is:

$$
\begin{aligned}
\left\langle\Xi^{V, \lambda} * \Xi^{V, \lambda} \mid \phi\right\rangle= & \lim _{m, n \rightarrow \infty}\left\langle\exp \left(-\lambda \int_{\frac{\pi}{4}}^{\left(\frac{m}{2}-\frac{1}{4}\right) \pi} V\left(x_{m+n-1}\right) d x_{m+n-1}\right)\right. \\
& \left.\exp \left(-\lambda \int_{\left(\frac{m}{2}-\frac{1}{4}\right) \pi}^{\left(\frac{n}{2}-\frac{1}{4}\right) \pi+\frac{1}{2}(m-1) \pi} V\left(x_{m+n-1}\right) d x_{m+n-1}\right) f \circ \phi(0)\right\rangle_{\widehat{C}_{m+n-1}} .
\end{aligned}
$$

This can be rewritten as

$$
\left\langle\Xi^{V, \lambda} * \Xi^{V, \lambda} \mid \phi\right\rangle=\lim _{m, n \rightarrow \infty}\left\langle\exp \left(-\lambda \int_{\frac{\pi}{4}}^{\frac{m+n-1}{2} \pi-\frac{\pi}{4}} V\left(x_{m+n-1}\right) d x_{m+n-1}\right) f \circ \phi(0)\right\rangle_{\widehat{C}_{m+n-1}} .
$$

This is precisely eq.(4.13) with $n$ replaced by $m+n-1$ (which is taken to infinity). This establishes the required result (4.15). 
In composing the integrals in passing to (4.17) the insertions of $V$ do coincide at one point, namely at $x_{m+n-1}=\left(\frac{m}{2}-\frac{1}{4}\right) \pi$. This will cause additional divergences even if the exponentials appearing in the definition of each $\Xi^{V, \lambda}$ are ultraviolet regularized. This divergence can be regulated as in subsection 4.1 by defining $\Xi^{V, \lambda}$ through eq.(4.13), with the $x_{n}$ integral running from $\frac{\pi}{4}+\epsilon$ to $\left(\frac{n}{2}-\frac{1}{4}\right) \pi-\epsilon$. Doing so, we miss in the exponent of eq.(4.17) an integral of $\lambda V$ over the range $\left(\left(\frac{m}{2}-\frac{1}{4}\right) \pi-\epsilon\right) \leq x_{m+n-1} \leq\left(\left(\frac{m}{2}-\frac{1}{4}\right) \pi+\epsilon\right)$ compared to the expression for $\left\langle\Xi^{V, \lambda} \mid \phi\right\rangle$. In the $m, n \rightarrow \infty$ limit this region moves off to infinity, and as a result contributes an $\epsilon$ dependent multiplicative factor to the correlation function given by the expectation value of the missing operator in the deformed theory. This is the analog of the factor of $(2 \epsilon)^{-2 h}$ which arose in the analysis of subsection 4.1, and can be absorbed in the definition of $\Xi^{V, \lambda}$ to ensure that it squares to itself under *-multiplication.

Since the operator $V$ is in the matter sector, $\left|\Xi^{V, \lambda}\right\rangle$ has the factorized form

$$
\left|\Xi^{V, \lambda}\right\rangle=\left|\Xi_{g}\right\rangle \otimes\left|\Xi_{m}^{V, \lambda}\right\rangle
$$

where $\Xi_{g}$ is independent of $V$ and $\lambda$. As before, with $\Xi_{g} *^{g} \Xi_{g}=\Xi_{g}$ we ensure that

$$
\Xi_{m}^{V, \lambda} *^{m} \Xi_{m}^{V, \lambda}=\Xi_{m}^{V, \lambda} .
$$

Thus we can now construct new D-brane solutions by taking the product $\left|\Psi_{g}\right\rangle \otimes\left|\Xi_{m}^{V, \lambda}\right\rangle$, where $\left|\Psi_{g}\right\rangle$ is the universal ghost state that appears in the D25-brane solution.

\subsection{Computation of the tension}

We would now like to compute the tension associated with this solution. This is proportional to $\left\langle\Xi_{m}^{V, \lambda} \mid \Xi_{m}^{V, \lambda}\right\rangle_{m}$. Computation of this inner product is again simple in the $\widehat{z}_{n}$ coordinates and the relevant geometry was discussed above (4.9). We therefore find

$$
\left\langle\Xi_{m}^{V, \lambda} \mid \Xi_{m}^{V, \lambda}\right\rangle_{m}=\lim _{m, n \rightarrow \infty}\left\langle\exp \left(-\lambda \int_{\frac{\pi}{4}}^{\frac{m+n-1}{2} \pi-\frac{\pi}{4}} V\left(x_{m+n-2}\right) d x_{m+n-2}\right)\right\rangle_{\widetilde{C}_{m+n-2}},
$$

where $x_{m+n-2}=\Re\left(z_{m+n-2}\right)$. If we do a more careful analysis using the regularized form of $\Xi^{V, \lambda}$, there will be two missing regions, each of width $2 \epsilon$, in the exponent of eq. (4.20). These regions are infinite distance apart in the $m, n \rightarrow \infty$ limit and each of

\footnotetext{
${ }^{10} \mathrm{We}$ are assuming here that the correlation function of the missing operator in the deformed theory satisfies cluster property.

${ }^{11}$ Since this computation involves matter sector fields only, there may be overall constant factors appearing at various stages due to non-zero central charge of the bulk matter theory. These are independent of $V$ and $\lambda$ and cancel when we compute the ratio of tensions.
} 
these regions give rise to an extra multiplicative factor, equal to the one that appeared in the computation of $\Xi^{V, \lambda} * \Xi^{V, \lambda}$, in eq.(4.20). These exactly cancel the $\epsilon$-dependent normalization of $\Xi^{V, \lambda}$ required to ensure that the regularized $\Xi^{V, \lambda}$ squares to itself under *-multiplication. This is analogous to the corresponding phenomenon in section 4.1.

We now define a rescaled coordinate $u$ as $u=4\left(z_{m+n-2}-\frac{\pi}{4}\right) /(m+n-2)$ so that $\Re(u)$ ranges from 0 to $2 \pi$. Thus in the $u$ coordinate we have a semiinfinite cylinder $C$ of circumference $2 \pi$. Writing $u=i \rho+\theta$, and taking into account the conformal transformation of the vertex operator $V$ under this scale transformation, we get:

$$
\left\langle\Xi_{m}^{V, \lambda} \mid \Xi_{m}^{V, \lambda}\right\rangle=\lim _{m, n \rightarrow \infty}\left\langle\exp \left(-\lambda_{R} \int_{\theta=0}^{2 \pi} d \theta V_{R}(\theta)\right)\right\rangle_{C}
$$

where $\lambda_{R} \int V_{R}$ denotes the operator to which the perturbation $\lambda \int V$ flows under the rescaling by $(m+n-2) / 4$. In particular, if $V$ has dimension $h$, it does not mix with other operators, and its conformal dimension remains unchanged under the renormalization group flow, then we have

$$
\lambda_{R} \int_{\theta=0}^{2 \pi} d \theta V_{R}(\theta)=\lambda\left(\frac{1}{4}(m+n-2)\right)^{1-h} \int_{\theta=0}^{2 \pi} d \theta V(\theta) .
$$

This semiinfinite cylinder in the $u$ coordinate is nothing but a unit disk $D_{U}$ with $\theta$ labeling the angular parameter along the boundary of the disk, and $e^{-\rho}$ labeling the radial coordinate. Thus what (4.21) represents is the partition function on a unit disk, with the perturbation $\lambda_{R} \int V_{R}(\theta) d \theta$ added at the boundary! Notice now that if $V$ is exactly marginal, i.e. if $h=1$ to all orders, then the perturbation is simply $-\lambda \int_{0}^{2 \pi} d \theta V(\theta)$, whereas if $V$ is a relevant deformation then $h<1$ and in the limit $m, n \rightarrow \infty, \lambda_{R} \int d \theta V_{R}(\theta)$ approaches its infrared fixed point $\lambda_{I R} \int d \theta V_{I R}(\theta)$. ⿷匚 Thus the net result is that $\left\langle\Xi_{m}^{V, \lambda} \mid \Xi_{m}^{V, \lambda}\right\rangle_{m}$ represents the partition function on the unit disk of the BCFT to which the theory flows in the infrared! As discussed below equation (4.10) this is indeed the tension of the D-brane associated to this BCFT. Thus we conclude that $\left|\Psi_{g}\right\rangle \otimes\left|\Xi_{m}^{V, \lambda}\right\rangle$ gives the D-brane solution corresponding to the BCFT to which the theory would flow in the infrared if we added to the action the boundary perturbation proportional to $\int V(\theta) d \theta$. We emphasize that the string field $\left|\Psi_{g}\right\rangle \otimes\left|\Xi_{m}^{V, \lambda}\right\rangle$ belongs to the state space of $\mathrm{BCFT}_{0}$.

In particular if we take $V$ to be the identity operator $I$, we see from eq.(4.13) that

$$
\left\langle\Xi^{I, \lambda} \mid \phi\right\rangle=\lim _{n \rightarrow \infty}\left\langle\exp \left(-\lambda \int_{\pi / 4}^{\left(\frac{n}{2}-\frac{1}{4}\right) \pi} d x_{n}\right) f \circ \phi(0)\right\rangle_{\widehat{C}_{n}}, \quad \forall|\phi\rangle \in \mathcal{H}
$$

\footnotetext{
${ }^{12}$ We shall not consider irrelevant perturbations as it is not clear how to tame the resulting ultraviolet divergences. Since they flow to zero in the IR, such perturbations are not expected to give rise to new solutions.
} 
For $\lambda>0$, this vanishes due to the infinite range of integration over $x_{n}$. Thus we get $\left|\Xi^{I, \lambda}\right\rangle=0$ and hence $\left|\Xi_{m}^{I, \lambda}\right\rangle=0$. This is consistent with the fact that the trivial solution $\Psi=0$ represents the tachyon vacuum configuration, and that in the boundary string field theory formalism, perturbation by the identity operator takes the unstable D-brane to the tachyon vacuum.

Note, however, that the solution (4.13) does seem to depend on $\lambda$ for more general relevant perturbations. Since different values of $\lambda$ correspond to the same tension of the final brane, we expect that they represent gauge equivalent solutions. Thus the parameter $\lambda$ is analogous to the redundant parameter $b$ labeling the lower dimensional D- $p$-brane solutions considered in ref. 30. [13

\subsection{Small deformations of the sliver}

From the analysis of subsection 4.3 it is clear that when the operator $V$ is relevant, the state $\left|\Xi^{V, \lambda}\right\rangle$ corresponds to a big change in field configuration since it gives rise to a totally new D-brane solution corresponding to the BCFT to which the theory flows in the infrared, whereas if $V$ is exactly marginal, then for small $\lambda,\left|\Xi^{V, \lambda}\right\rangle$ is a solution 'close to' the original solution $\Xi$. Thus we can define a small deformation $\Delta^{V}$ around the solution $\Xi$ through the relation:

$$
\Xi^{V, \lambda}=\Xi+\lambda \Delta \Xi^{V}+O\left(\lambda^{2}\right)
$$

and using eqs.(4.13) and (4.14) we find

$$
\begin{aligned}
\left\langle\Delta \Xi^{V} \mid \phi\right\rangle & =-\lim _{n \rightarrow \infty}\left\langle\int_{\frac{\pi}{4}}^{\left(\frac{n}{2}-\frac{1}{4}\right) \pi} V\left(x_{n}\right) d x_{n} f \circ \phi(0)\right\rangle_{\widehat{C}_{n}} \\
& =-\left\langle\int_{\left|x^{\prime}\right|>\frac{\pi}{4}} V\left(x^{\prime}\right) d x^{\prime} f \circ \phi(0)\right\rangle_{D_{H}}, \quad \forall|\phi\rangle \in \mathcal{H} .
\end{aligned}
$$

By expanding eq.(4.15) in powers of $\lambda$, it follows that $\Delta \Xi^{V}$ satisfies

$$
\Xi * \Delta \Xi^{V}+\Delta \Xi^{V} * \Xi=\Delta \Xi^{V} \text {. }
$$

Thus $\left|\Psi_{g}\right\rangle \otimes\left|\Delta \Xi_{m}^{V}\right\rangle$ will describe small deformation of the string field theory solution describing the D-brane corresponding to $\mathrm{BCFT}_{0}$. One can also give a direct proof of eq. 4.26) by using the $*$-product rules and the gluing relations (3.35) as follows. For this

\footnotetext{
${ }^{13}$ This was suggested to us by Witten [50].

${ }^{14}$ Even though $\left\langle\Xi_{m} \mid \Xi_{m}^{V, \lambda}\right\rangle_{m}$ vanishes even for small $\lambda$, we treat the solutions as 'close' in the sense that the BCFT's corresponding to $\lambda=0$ and $\lambda$ small have correlation functions which differ from each other by small amount.
} 
we shall represent both $\Delta \Xi^{V}$ through the first of eq.4.25) and $\Xi$ by a similar formula without the $\int V\left(x_{n}\right) d x_{n}$ insertion for a fixed finite $n$ and then take $n \rightarrow \infty$ limit at the end of the calculation. Using the by now standard procedure we get:

$$
\begin{aligned}
& \left\langle\Delta \Xi^{V} * \Xi \mid \phi\right\rangle=-\lim _{n \rightarrow \infty}\left\langle\int_{\frac{\pi}{4}}^{\pi\left(\frac{n}{2}-\frac{1}{4}\right)} d x_{2 n-1} V\left(x_{2 n-1}\right) f \circ \phi(0)\right\rangle_{\widehat{C}_{2 n-1}} \\
& \left\langle\Xi * \Delta \Xi^{V} \mid \phi\right\rangle=-\lim _{n \rightarrow \infty}\left\langle\int_{\pi\left(\frac{n}{2}-\frac{1}{4}\right)}^{\pi\left(n-\frac{3}{4}\right)} d x_{2 n-1} V\left(x_{2 n-1}\right) f \circ \phi(0)\right\rangle_{\widehat{C}_{2 n-1}} .
\end{aligned}
$$

Thus we have:

$$
\left\langle\Delta \Xi^{V} * \Xi \mid \phi\right\rangle+\left\langle\Xi * \Delta \Xi^{V} \mid \phi\right\rangle=-\lim _{n \rightarrow \infty}\left\langle\int_{\frac{\pi}{4}}^{\pi\left(n-\frac{3}{4}\right)} d x_{2 n-1} V\left(x_{2 n-1}\right) f \circ \phi(0)\right\rangle_{\widehat{C}_{2 n-1}} .
$$

Introducing $m=2 n-1$ we can rewrite this as

$$
\left\langle\Delta \Xi^{V} * \Xi \mid \phi\right\rangle+\left\langle\Xi * \Delta \Xi^{V} \mid \phi\right\rangle=-\lim _{m \rightarrow \infty}\left\langle\int_{\frac{\pi}{4}}^{\pi\left(\frac{m}{2}-\frac{1}{4}\right)} d x_{m} V\left(x_{m}\right) f \circ \phi(0)\right\rangle_{\widehat{C}_{m}} .
$$

This is precisely the expression (4.25) for $\left\langle\Delta \Xi^{V} \mid \phi\right\rangle$. Thus we see that $\Delta \Xi^{V}$ satisfies equation (4.26).

For later use we need to use a regularized expression for $\Delta \Xi^{V}$ by restricting the integration range in (4.25) to be $\left|x^{\prime}\right| \geq \frac{\pi}{4}+\epsilon$, or equivalently, $\frac{\pi}{4}+\epsilon \leq x_{n} \leq\left(\frac{n}{2}-\frac{1}{4}\right) \pi-\epsilon$. When we compute the analog of (4.28) with this regularized expression for $\Delta \Xi^{V}$, and compare this with the regularized version of (4.25), we find that we miss an integral

$$
-\int_{\left(\frac{n}{2}-\frac{1}{4}\right) \pi-\epsilon}^{\left(\frac{n}{2}-\frac{1}{4}\right) \pi+\epsilon} d x_{2 n-1}\left\langle V\left(x_{2 n-1}\right) f \circ \phi(0)\right\rangle_{\widehat{C}_{2 n-1}} .
$$

In the $n \rightarrow \infty$ limit the integration region moves off to infinity and the correlator above vanishes as long as $V$ has dimension $>0$. Thus the regularized $\Delta \Xi^{V}$ still satisfies eq.(4.26). In the next subsection we shall interpret $\Delta \Xi^{V}$ as an appropriate covariant derivative of the surface state along a marginal direction in the space of boundary conformal field theories.

\subsection{Background independence and theory-space connections}

The string field theory action around the tachyon vacuum enjoys a fundamental property: it has manifest background independence with respect to the open string moduli. This observation will allow us to recover the construction of infinitesimal deformations of classical solutions from the point of view of deformations along marginal directions in the open string theory moduli space. To explain these ideas, we need to recall some notions 
about connections in theory space. Our discussion will follow [51, 55], with some obvious modifications needed for open strings.

Let us fix the CFT in the bulk (the closed string moduli) and for this given bulk CFT, let us consider the space of BCFT's, labeled by some continuous parameters $\left\{x^{s}\right\}$. Each point $\left\{x^{s}\right\}$ specifies a BCFT, with its own state space $\mathcal{H}_{x}$. We have the structure of a vector bundle, where the base is theory space (parameterized by $\left\{x^{s}\right\}$ ), and the fiber at each point $x$ is $\mathcal{H}_{x}$. Thus we can introduce connections on this vector bundle. While there are many possible connections, a natural choice for open string field theory is the canonical connection $\widehat{\Gamma}[51]$. On surface states, the associated covariant derivative $\widehat{D}_{s}$ acts as the integration of the exactly marginal perturbation $\mathcal{O}_{s}$ over the boundary of the Riemann surface, excluding from the integration region the segments around the punctures where local coordinates are defined:

$$
\widehat{D}_{s}\langle\Sigma|=-\int_{\partial \Sigma-\cup_{i} D_{i}} d z^{\prime}\left\langle\Sigma ; z^{\prime} \mid \mathcal{O}_{s}\right\rangle
$$

Here $\Sigma$ is a disk with $n$ punctures on its boundary $\partial \Sigma$ with some global coordinate $z$. The regions $D_{i} \subset \partial \Sigma$ are the images of the diameters of local coordinates half-disks $\left\{\left|\xi_{i}\right| \leq 1, \Im(\xi)=0\right\}$. The surface state $\left\langle\Sigma ; z^{\prime}\right|$ is the $n+1$-punctured disk obtained by introducing an extra puncture on $\Sigma$ at the boundary point $z=z^{\prime}$, with local coordinate $\xi=z-z^{\prime}$. Finally $\mathcal{O}_{s}$ is an exactly marginal operator of the boundary CFT inserted at this new puncture.

The reflector state ${ }_{12}\langle R|$ and the cubic vertex ${ }_{123}\left\langle V_{3}\right|$ are covariantly constant with respect to this connection:

$$
\widehat{D}_{s 12}\langle R|=0, \quad \widehat{D}_{s 123}\left\langle V_{3}\right|=0 \text {. }
$$

This follows immediately from the fact that the local coordinate patches cover completely the two and three punctured disks associated to ${ }_{12}\langle R|$ and ${ }_{123}\left\langle V_{3}\right|$, so that in both cases $\partial \Sigma-\cup_{i} D_{i}$ is the empty set. We can rephrase (4.32) by saying that $\widehat{D}_{s}$ acts as a derivation of the $*$ product:

$$
\widehat{D}_{s}\left(\phi_{1} * \phi_{2}\right)=\widehat{D}_{s} \phi_{1} * \phi_{2}+\phi_{1} * \widehat{D}_{s} \phi_{2}
$$

where $\phi_{1}$ and $\phi_{2}$ are two sections of the vector bundle.

At each point $x$ in theory space, exactly marginal deformations are represented by dimension one primaries $\mathcal{O}_{s}$ of the matter BCFT. Thus for each exactly marginal direction

\footnotetext{
${ }^{15}$ The reflector ${ }_{12}\langle R|$ is the two-punctured disk with punctures at $z=0$ and $z=\infty$ in UHP coordinates, with local coordinates $\xi_{1}=z$ around $z=0$ and $\xi_{2}=-1 / z$ around $z=\infty$. The reflector provides the definition of the BPZ inner product: $\langle\psi \mid \phi\rangle \equiv{ }_{12}\langle R \mid \psi\rangle_{1}|\phi\rangle_{2}$.
} 
$s$, the covariant derivative $\widehat{D}_{s}$ does not involve any ghosts and commutes with the purely ghost kinetic operator $\mathcal{Q}$ of vacuum string field theory,

$$
\left[\widehat{D}_{s}, \mathcal{Q}\right]=0
$$

The string field action (2.1) is naturally a function on the vector bundle. $\mathcal{S}(\Psi)$ is indeed $\mathcal{S}\left(\psi^{i}, x\right)$ where $\psi^{i}$ are components along the vectors in $\mathcal{H}_{x}$. It can be written as

$$
\mathcal{S}(\Psi, x) \equiv-\frac{1}{g_{0}^{2}}\left[\frac{1}{2}\langle R \mid \Psi\rangle \mathcal{Q}|\Psi\rangle+\frac{1}{3}\left\langle V_{3} \mid \Psi\right\rangle|\Psi\rangle|\Psi\rangle\right]
$$

It follows from the definition of a covariant derivative of functions on a vector bundle (such derivative measures how the function changes as we move on the base, and on the fiber by parallel transport) and relations (4.32) and (4.34) that

$$
\widehat{D}_{s} \mathcal{S}=0 \text {. }
$$

This is the statement of manifest background independence of the vacuum string field theory: there is a (canonical) connection $\widehat{\Gamma}$ so that the action is covariantly constant. This means that vacuum string field theory is independent of the choice of the reference boundary conformal field theory $\mathrm{BCFT}_{0}$ - string field actions using nearby $\mathrm{BCFT}^{\prime}$ s are identical when the string fields are related by an homogeneous field redefinition generated by parallel transport with the connection $\widehat{\Gamma}$. This is simpler than for conventional string field theory [55], where the covariant derivative of the action is nonvanishing and the requisite field redefinition includes a constant shift and other terms in addition to parallel transport.

Consider now a solution $\Psi^{x}=\Psi_{g} \otimes \Psi_{m}^{x}$ of the vacuum string field theory equations corresponding to a certain BCFT background labeled by $x$, expressed as an element of $\mathcal{H}_{x} .\left|\Psi_{m}^{x}\right\rangle$ is simply the matter part of the sliver state $\left|\Xi_{m}^{x}\right\rangle$ associated with this conformal field theory. Thus we have

$$
\mathcal{Q} \Psi^{x}+\Psi^{x} * \Psi^{x}=0, \quad \Psi_{m}^{x} *^{m} \Psi_{m}^{x}=\Psi_{m}^{x}
$$

Since $\Psi^{x}$ is defined as an element of $\mathcal{H}_{x}$ for every $x$, we have a section of the vector bundle introduced earlier. Using the fact that $\widehat{D}_{s}\left|\Psi_{g}\right\rangle=0$, we have

$$
\widehat{D}_{s}\left|\Psi^{x}\right\rangle=\left|\Psi_{g}\right\rangle \otimes \widehat{D}_{s}\left|\Xi_{m}^{x}\right\rangle
$$

Since $\left|\Xi_{m}^{x}\right\rangle$ is a surface state, its covariant derivative is given by eq.(4.31). Comparing this with (4.25) we see that this is just $\left|\Delta \Psi^{\mathcal{O}_{s}}\right\rangle$. Thus we have

$$
\widehat{D}_{s}\left|\Psi^{x}\right\rangle=\left|\Psi_{g}\right\rangle \otimes\left|\Delta \Psi^{\mathcal{O}_{s}}\right\rangle .
$$


The small deformation of the sliver solution considered in the previous subsection is simply the covariant derivative of $\Psi^{x}$ with the connection $\widehat{\Gamma}$. Indeed, applying $\widehat{D}_{s}$ on the second of eq.(4.37), and using eqs.(4.33), one gets

$$
\left(\widehat{D}_{s} \Psi_{m}^{x}\right) *^{m} \Psi_{m}^{x}+\Psi_{m}^{x} *^{m}\left(\widehat{D}_{s} \Psi_{m}^{x}\right)=\widehat{D}_{s} \Psi_{m}^{x} .
$$

This shows that $\widehat{D}_{s} \Psi^{x}$ satisfies the small fluctuation equation (4.26).

Although we have been careful in phrasing the discussion in terms of exactly marginal deformations, as only for those it makes sense to talk about connections in theory space, for infinitesimal deformations we can nevertheless consider the generalization that $\mathcal{O}_{s}$ is a generic dimension one matter primary. The operator $\widehat{D}_{s}$ is still well-defined and obeys (4.33) and (4.34). So we obtain a solution $\widehat{D}_{s} \Psi^{x}$ of the small fluctuation equations for each dimension one matter primary. These states are promising candidates for the spectrum of physical excitations around the D-brane background. We shall discuss this in detail in section 5 .

It is intriguing that some of the previous analysis appears to apply formally to closed string deformations. A closed string deformation of a surface state is obtained by integrating a closed string marginal operator $\mathcal{O}_{\bar{s}}$ over the bulk of the surface state, leaving out the local coordinate regions. It would seem that such deformation of the sliver would satisfy the small fluctuation equation. In this case, however, this integral is plagued with ultraviolet divergences from the region of integration where the operator $\mathcal{O}_{\bar{s}}$ comes close to the boundary of the disk, and it is not a priori clear how such divergences should be regulated. This deformation - if it can be defined - might just be the open string byproduct of a shift in the closed string background, as is possibly the case in open/closed string field theory [62]. But perhaps it represents some true encoding of closed string physics into the open string state space. It is worth noting that the sliver, as opposed to the identity string field used earlier for encoding closed string deformations in open string theory [63], has plenty of bulk to insert closed string states. Clearly this issue deserves further investigation.

\section{Physical states around D-brane backgrounds}

Given a candidate solution representing a specific D-brane, one would like to find the spectrum of physical states around the D-brane background and compare this with the known spectrum of physical open string states in that particular D-brane background. Let $\Psi_{0}=\Psi_{g} \otimes \Psi_{m}$ be a solution representing a particular D-brane in the string field theory around the tachyon vacuum [30]. After shifting the string field $\Psi=\Psi_{0}+\Phi$, the 
action takes the form:

$$
\mathcal{S}(\Phi) \equiv-\frac{1}{g_{0}^{2}}\left[\frac{1}{2}\left\langle\Phi, \mathcal{Q}_{0} \Phi\right\rangle+\frac{1}{3}\langle\Phi, \Phi * \Phi\rangle\right]
$$

where the action of the BRST operator $\mathcal{Q}_{0}$ in the new background on any state $|A\rangle$ in $\mathcal{H}$ is given as

$$
\mathcal{Q}_{0} A=\mathcal{Q} A+\Psi_{0} * A-(-)^{A} A * \Psi_{0} .
$$

Around the new background the physical state condition, corresponding to the linearized equations of motion, is then

$$
\mathcal{Q}_{0} \Phi=\mathcal{Q} \Phi+\Psi_{0} * \Phi+\Phi * \Psi_{0}=0
$$

while the linearized gauge symmetries are given as

$$
\delta_{\Lambda} \Phi=\mathcal{Q}_{0} \Lambda=\mathcal{Q} \Lambda+\Psi_{0} * \Lambda-\Lambda * \Psi_{0} .
$$

Physical states around $\Psi_{0}$ are identified with the cohomology classes of $\mathcal{Q}_{0}$.

Clearly a full solution of this problem requires the knowledge of $\mathcal{Q}$, and the solution $\Psi_{0}$ including its ghost part, which are not available to us at present. In this section we shall try to get some insight into this problem.

\subsection{Factorization ansatz for fluctuations}

Clearly there are two parts to the problem. First we need to find solutions to the linearized equations of motion (5.3) and then we need to determine which of these are related by gauge transformations. For the first problem, we could look at a subset of field configurations of the form:

$$
\Phi=\Psi_{g} \otimes \delta \Psi_{m},
$$

where $\delta \Psi_{m}$ is a matter state. Replacing (5.5) into (5.3) and using eq.(2.6) we find the condition

$$
\Psi_{m} *^{m} \delta \Psi_{m}+\delta \Psi_{m} *^{m} \Psi_{m}=\delta \Psi_{m} .
$$

Note that this condition does not involve the operator $\mathcal{Q}$. Clearly not every solution of (5.3) is of the form (5.5). However it is conceivable that every solution of (5.3) can be brought to this form using a gauge transformation (5.4), i.e. there is a representative element of the form (5.5) in every cohomology class of $\mathcal{Q}_{0}$.

It is possible to find solutions to eq.(5.6). For definiteness we shall take $\Psi_{m}$ to be $\Xi_{m}$, - the matter part of the sliver state associated with $\mathrm{BCFT}_{0}$, - and suppose that it represents a particular D-brane in a moduli space that includes some marginal directions. 
As discussed in section 1 , the various points in this moduli space can be obtained as deformed sliver solutions. Defining $\Delta \Xi_{m}^{V}$ as the matter part of $\Delta \Xi^{V}$ defined in section 4.5 and using eq.(4.26) we see that $\delta \Psi_{m} \propto \Delta \Xi_{m}^{V}$ satisfies eq.(5.6). Although the argument given above uses an exactly marginal deformation, the explicit analysis of section 4.5, showing that $\Delta \Xi^{V}$ satisfied eq.(4.26), goes through if we choose $V$ to be an arbitrary dimension one primary operator. As a result $\delta \Psi_{m} \propto \Delta \Xi_{m}^{V}$ still satisfies eq.(5.6). Thus we seem to have gotten a solution of the linearized equations of motion for every dimension one matter primary operator in $\mathrm{BCFT}_{0}$.

While this is encouraging, there are some subtleties which we now discuss. First of all, we compute the inner product $\left\langle\Delta \Xi_{m}^{V} \mid \Delta \Xi_{m}^{V}\right\rangle$ as in eq.4.20). This is given by:

$$
\begin{aligned}
\left\langle\Delta \Xi_{m}^{V} \mid \Delta \Xi_{m}^{V}\right\rangle= & \lim _{m, n \rightarrow \infty}\left\langle\left(\int_{\frac{\pi}{4}}^{\left(\frac{m}{2}-\frac{1}{4}\right) \pi} V\left(x_{m+n-2}\right) d x_{m+n-2}\right)\right. \\
& \left.\left(\int_{\left(\frac{m}{2}-\frac{1}{4}\right) \pi}^{\frac{m+n-1}{2} \pi-\frac{\pi}{4}} V\left(x_{m+n-2}\right) d x_{m+n-2}\right)\right\rangle_{\widetilde{C}_{m+n-2}} .
\end{aligned}
$$

The correlation functions are computed in the matter sector only. In the $m, n \rightarrow \infty$ limit the range of integration over $x_{m+n-2}$ is infinite, but this is easily avoided by going to a new coordinate system $u \equiv i \rho+\theta=4\left(z_{m+n-2}-\pi / 4\right) /(m+n-2)$ which maps $\widetilde{C}_{m+n-2}$ to a seminfinite cylinder $C$ of circumference $2 \pi$. Dimension one primary fields will not pick up any factor under this rescaling, and we get

$$
\left\langle\Delta \Xi_{m}^{V} \mid \Delta \Xi_{m}^{V}\right\rangle=\left\langle\left(\int_{0}^{\pi} V(\theta) d \theta\right)\left(\int_{\pi}^{2 \pi} V\left(\theta^{\prime}\right) d \theta^{\prime}\right)\right\rangle_{C} .
$$

Note now that the range of integration over $\theta$ and $\theta^{\prime}$ coincide at two points, $\theta=0$ and $\theta=\pi$. Since $\left\langle V(\theta) V\left(\theta^{\prime}\right)\right\rangle \sim\left|\theta-\theta^{\prime}\right|^{-2}$ for small $\left|\theta-\theta^{\prime}\right|$, this gives logarithmically divergent integrals. Thus the BPZ norm of $\Delta \Xi_{m}^{V}$ in the matter sector is logarithmically divergent. This of course is related to the ultraviolet divergence discussed in section 4 and one could try to regularize this by taking the limit of $x^{\prime}$ integration in eq.(4.25) to be in the range $\left|x^{\prime}\right| \geq \frac{\pi}{4}+\epsilon$. As discussed in section 4.5, $\Delta \Xi^{V}$ satisfies eq. (4.26) even when $\epsilon$ is finite. However unlike $\Xi_{m}^{V, \lambda}$, which has finite norm for finite $\epsilon$ (when measured with respect to the norm of $\left.\Xi_{m}\right), \Delta \Xi_{m}^{V}$ has infinite norm even for finite $\epsilon$. To see this we note that the rescaling needed to go from the $x_{m+n-2}$ coordinate to the $\theta$ coordinate shrinks a region of finite width to zero width. More precisely, with the regulator $\epsilon$ in place and after the rescaling, we get

$$
\left\langle\Delta \Xi_{m}^{V} \mid \Delta \Xi_{m}^{V}\right\rangle=\lim _{m, n \rightarrow \infty}\left\langle\left(\int_{4 \epsilon /(m+n-2)}^{\pi-4 \epsilon /(m+n-2)} V(\theta) d \theta\right)\left(\int_{\pi+4 \epsilon /(m+n-2)}^{2 \pi-4 \epsilon /(m+n-2)} V\left(\theta^{\prime}\right) d \theta^{\prime}\right)\right\rangle_{C} .
$$

Thus even for finite $\epsilon$ the integral has logarithmic divergences in the $m, n \rightarrow \infty$ limit, since the integration limits approach each other. This divergence is actually needed for 
consistency. Indeed, note that for a marginal deformation $\Xi_{m}^{V, \lambda}$ can be identified with $\Xi_{m}^{\prime}$ associated with a deformed $\mathrm{BCFT}^{\prime}$ discussed in section 4.1. The norm of $\Xi_{m}^{\prime}$, being equal to $(2 \epsilon)^{-4 h}$ times the partition function of $\mathrm{BCFT}^{\prime}$ on the cylinder, approaches that of $\Xi_{m}$ for small $\lambda$ since as $\lambda \rightarrow 0, h \rightarrow 0$ and the partition functions of $\mathrm{BCFT}^{\prime}$ and $\mathrm{BCFT}_{0}$ are equal. The orthonormality of $\Xi_{m}$ and $\Xi_{m}^{V, \lambda}=\Xi_{m}^{\prime}$ (section (4.1)) then leads to the result that the norm of $\left(\Xi_{m}^{V, \lambda}-\Xi_{m}\right)$ is twice the norm of $\Xi_{m}$ in the $\lambda \rightarrow 0$ limit. Given that $\Xi_{m}^{V, \lambda}=\Xi_{m}+\lambda \Delta \Xi_{m}^{V}+\mathcal{O}\left(\lambda^{2}\right)$, we reach the conclusion that $\lambda^{2}$ times the norm of $\Delta \Xi_{m}^{V}$ is twice the norm of $\Xi_{m}$ in the small $\lambda$ limit. If this has to be true for arbitrarily small $\lambda$, then $\Delta \Xi_{m}^{V}$ must have infinite norm.

This divergence is not necessarily problematic, since it is not clear a priori that having finite norm in the matter sector is a requirement on physical deformations. As we have seen in section (4.1), the deformed solution $\Xi_{m}^{\prime}$ does have finite norm, even if $\Delta \Xi_{m}^{V}$ has divergent norm. We shall also argue later that having a logarithmically divergent norm of this kind may be a necessary condition for a physical deformation not to be pure gauge. From a physical viewpoint, since we have seen that for exactly marginal $V, \Delta \Xi^{V}$ represents some small deformation of $\mathrm{BCFT}_{0}$, we are led to believe that such deformations must be allowed in the computation of the spectrum.

The second subtlety arises because the manipulations in section 4.5 leading to eq.(4.26) actually hold without any constraint on $V$ as the gluing in the $\widehat{z}_{n}$ coordinate system does not require us to make any conformal transformations at all. So one might ask: why can't we use the matter part of these states to find new solutions of eq.(5.6)? The answer to this question may again be hidden in the normalization of the state. For this, let us write down the norm of $\Delta \Xi_{m}^{V}$ for an operator $V$ of arbitrary dimension $h$ :

$$
\begin{aligned}
\left\langle\Delta \Xi_{m}^{V} \mid \Delta \Xi_{m}^{V}\right\rangle= & \lim _{m, n \rightarrow \infty}\left[( \frac { m + n - 2 } { 4 } ) ^ { 2 ( 1 - h ) } \left\langle\left(\int_{4 \epsilon /(m+n-2)}^{\pi-4 \epsilon /(m+n-2)} V(\theta) d \theta\right)\right.\right. \\
& \left.\left.\left(\int_{\pi+4 \epsilon /(m+n-2)}^{2 \pi-4 \epsilon /(m+n-2)} V\left(\theta^{\prime}\right) d \theta^{\prime}\right)\right\rangle_{C}\right] .
\end{aligned}
$$

Note the prefactor coming from the conformal transformation of the vertex operators when we make the final conversion from $x_{m+n-2}$ to $\theta$ coordinate. For $h<1$ there is no short distance divergence from the limits of integration of $\theta, \theta^{\prime}$, but the contribution from the finite $\theta$ region has power law divergence due to the infinite prefactor in the $m, n \rightarrow \infty$ limit. Thus the divergence in the norm of such states is worse than that of a marginal deformation, and clearly originates from the large distance region (in the original $x_{m+n-2}$ coordinate system.) In fact this divergence is precisely the reason why a small perturbation by $V$ drives the system all the way to the infrared fixed point. Thus we can conclude with reasonable degree of confidence that for $h<1$ the $\Delta \Xi^{V}$ does not represent small deformation. On the other hand for $h>1$ the integral is finite for finite 
$\epsilon$ as the short distance singularity coming from the region of integration $\theta \simeq \theta^{\prime}$ at 0 or $\pi$ cancel the conformal factor exactly. Thus $\Delta \Xi^{V}$ has finite norm. This seems to make irrelevant perturbations better behaved. As we shall see below, however, these are most likely pure gauge deformations, precisely because they have finite norm.

\subsection{Factorization ansatz for gauge transformations}

Not all solutions of (5.6) represent inequivalent physical states, since we still need to consider the linearized gauge invariances (5.4). A gauge transformation (5.4) with generic parameter $\Lambda$ will not respect the form (5.5) of the fluctuations. While there may be more general gauge parameters respecting (5.5) we now restrict ourselves to factored gauge parameters $\Lambda=\Lambda_{g} \otimes \Lambda_{m}$, where $\Lambda_{m}$ is a matter state and $\Lambda_{g}$ is a ghost state satisfying

$$
\mathcal{Q} \Lambda_{g}=0, \quad \Lambda_{g} *^{g} \Psi_{g}=\Psi_{g} *^{g} \Lambda_{g}=\Psi_{g}
$$

For such a $\Lambda_{g}$, (5.4) gives

$$
\begin{aligned}
\delta_{\Lambda}(\Phi) & =\mathcal{Q} \Lambda_{g} \otimes \Lambda_{m}+\left(\Psi_{g} *^{g} \Lambda_{g}\right) \otimes\left(\Xi_{m} *^{m} \Lambda_{m}-\Lambda_{m} *^{m} \Xi_{m}\right) \\
\rightarrow \Psi_{g} \otimes \delta_{\Lambda} \delta \Psi_{m} & =\Psi_{g} \otimes\left(\Xi_{m} *^{m} \Lambda_{m}-\Lambda_{m} *^{m} \Xi_{m}\right) .
\end{aligned}
$$

It follows from this equation and from (5.6) that we can study physical states around D-brane backgrounds by considering the space of equivalence classes $\mathcal{H}_{\delta \Psi}$ of matter string fields $\delta \Psi_{m}$ which satisfy the equations:

$$
\begin{aligned}
& \Xi_{m} *^{m} \delta \Psi_{m}+\delta \Psi_{m} *^{m} \Xi_{m}=\delta \Psi_{m} \\
& \delta \Psi_{m} \simeq \delta \Psi_{m}+\Xi_{m} *^{m} \Lambda_{m}-\Lambda_{m} *^{m} \Xi_{m} .
\end{aligned}
$$

We should keep in mind, however, that there may be gauge transformation parameters other than those of the form $\Lambda_{g} \otimes \Lambda_{m}$ which generate factorized deformations of the form $\Psi_{g} \otimes \delta \Psi_{m}$. These will generate further equivalence relations between the deformations $\delta \Psi_{m}$.

Does a $\Lambda_{g}$ satisfying (5.11) exist? Let $\mathcal{I}_{g}$ denote the ghost part of the identity string state, normalized so that $\mathcal{I}_{g} *^{g} \mathcal{I}_{g}=\mathcal{I}_{g}$. If $\mathcal{Q}$ annihilates the identity state, then $\Lambda_{g}=\mathcal{I}_{g}$ clearly satisfies eq.(5.11). We shall now argue that $\Lambda_{g}=\mathcal{I}_{g}$ is also the most natural choice. From the analysis of refs. [56, 57, 58, 59, 60, 31, 49, 61] we know that barring the complications of the string mid-point coordinate and/or zero modes, it is natural to think of the string field as a matrix acting on the half string state space. In particular we can think of the factored string field of the form (2.5) as the direct product of two matrices, one acting on the ghost sector of the half-string state and the other acting on the matter 
sector of the half-string state space. Since the identity string field $\mathcal{I}$ can be regarded as an identity matrix on the half string state space, choosing $\Lambda=\mathcal{I}_{g} \otimes \Lambda_{m}$ corresponds to a matrix which is a direct product of the identity matrix in the ghost sector of the half-string state space and generator of a non-trivial rotation in the matter sector of the half-string state space. This is precisely how we would represent the rotation generator on a product of two vector spaces if we want to construct generators that act on only one of the two vector spaces. Thus from this physical point of view choosing $\Lambda_{g}=\mathcal{I}_{g}$ would be most natural. This in turn, would require us to choose $\mathcal{Q}$ that annihilates $\mathcal{I}_{g}$. We take this as a strong argument in favor of choosing such $\mathcal{Q}$ Given this choice we can now proceed by taking (5.14) to be a valid gauge equivalence relation between different $\delta \Psi_{m}$.

Since these equations refer to matter fluctuations $\delta \Psi_{m}$, without risk of confusion we shall from now on drop all $m$ subscripts and superscripts referring to matter and write these equations as

$$
\begin{aligned}
& \Xi * \delta \Psi+\delta \Psi * \Xi=\delta \Psi \\
& \delta \Psi \simeq \delta \Psi+\Xi * \Lambda-\Lambda * \Xi .
\end{aligned}
$$

In the next two subsections we discuss some consequences of these equations. All states, *-products and correlation functions in these subsections will refer to matter sector only.

\subsection{Deformed projectors and rules for physical states}

The physical state conditions (5.15) obtained above have the interpretation of deformation equations for projectors, with a familiar equivalence relation. Indeed this equation is clearly equivalent to the condition that $(\Xi+\delta \Psi)$ be a projector:

$$
(\Xi+\delta \Psi) *(\Xi+\delta \Psi)=(\Xi+\delta \Psi)+\mathcal{O}\left(\delta \Psi^{2}\right)
$$

Similarly, equation (5.16) is equivalent to

$$
\Xi+\delta \Psi \simeq e^{-\Lambda} * \Xi * e^{\Lambda}+\mathcal{O}\left(\Lambda^{2}\right)
$$

where in the expansion of $e^{\Lambda}$ products correspond to $*$-products. Therefore our physical state conditions are conditions for deformed projectors with a $U(\infty)$ local gauge symmetry.

This leads to a puzzle. It is simple to show formally that the above physical state problem has no nontrivial solutions! Indeed, we multiply (5.15) from the left by $\Xi$ and use associativity and the projector condition for $\Xi$ to obtain

$$
\Xi * \delta \Psi * \Xi=0
$$

\footnotetext{
${ }^{16}$ The result of ref. [24], showing that around the tachyon vacuum $\mathcal{I}$ is a pure gauge state, also requires us to choose such a $\mathcal{Q}$.
} 
It then suffices to take

$$
\Lambda=\Xi * \delta \Psi-\delta \Psi * \Xi
$$

to verify that

$$
\begin{aligned}
\Xi * \Lambda-\Lambda * \Xi & =\Xi * \delta \Psi-2 \Xi * \delta \Psi * \Xi+\delta \Psi * \Xi \\
& =\Xi * \delta \Psi+\delta \Psi * \Xi=\delta \Psi
\end{aligned}
$$

where we used associativity, equation (5.19) and (5.15). It follows that $\delta \Psi$ is trivial.

This clearly shows that if eq.(5.16) is the correct equivalence relation, we cannot get non-trivial elements of the cohomology using states which are completely regular. One way to avoid this problem is to use a non-normalizable $\delta \Psi$. This will typically give rise to non-normalizable $\Lambda$ of the same type via eq.(5.20). If the requirement of normalizability on string field fluctuations is different from that on the gauge transformation parameters (which is not improbable considering that the two are multiplied by different ghost sector states) it is possible to have a non-normalizable $\delta \Psi$ which is an allowed deformation, while the corresponding $\Lambda$ constructed through eq.(5.20) is not an allowed gauge transformation parameter.

Indeed, this suggestion is physically motivated. We have argued earlier that despite having logarithmically divergent norms, deformations $\delta \Psi \propto \Delta \Xi_{m}^{V}$ associated with marginal operators $V$ should be considered as physical deformations. It is easy to verify that $\Lambda$ constructed from such a $\delta \Psi$ through eq.(5.20) also has logarithmically divergent norm. If such non-normalizable $\Lambda$ 's did generate gauge transformations, then we would be forced to conclude that two different BCFT's related by a marginal deformation are gauge equivalent. Since this is not physically acceptable, we are led to the conclusion that matter sector states $\Lambda$ with logarithmically divergent norms are not valid gauge transformation parameters. This principle will also explain why the state associated with deformations by irrelevant operators, found at the end of section 5.1, are pure gauge; they have finite norm and the corresponding gauge transformation parameter $\Lambda$ defined through eq.(5.20) also has finite norm. Thus such deformations can be gauged away.

While this principle leads us to identify deformations of the sliver by dimension-one primaries as physical states, it also includes in the list deformations by unwanted dimensionone operators - the vertex operators of null states and non-primary states. At present it is not clear how to eliminate them and reproduce the correct spectrum of physical states on the D-brane.円

\footnotetext{
${ }^{17}$ Although a null state has vanishing two point function with itself, its two point function with a vertex operator which is neither a primary nor a secondary, could be non-zero.
} 


\section{Discussion}

In this concluding section we make some general remarks about the string field theory action given in eq.(2.1) and also discuss the implication of the results of the present paper in a general context. We end with a discussion of some of the open problems in this subject.

- Typically the formulation of cubic open string field theory requires a choice of background, determining the form of the quadratic term in the action. In that sense the action (2.1) represents the choice of the tachyon vacuum as the background around which we expand. But this clearly is a special background being the end-point of tachyon condensation of any D-brane. The action is formally independent of the choice of BCFT whose basis we use to expand the string field, as is apparent from the fact that $\mathcal{Q}$ is made purely of ghost operators, and the $*$-product, defined through overlap conditions on string wave-functionals, is formally independent of the choice of open string background. At least for backgrounds related by exactly marginal deformations, this notion of manifest background independence can be made precise using the language of connections in theory space [51, 55], as we demonstrated in section 4.6. Starting from the tachyon vacuum we can reach any D-brane configuration by simply considering the sliver associated with that particular BCFT. Note, however, that the spirit here is somewhat different from that of boundary string field theory, - in our approach we always represent the string field as an element of the state space of a specific reference BCFT. At the end it should not matter which basis we use to expand the string field. Indeed, we have seen in section that physical quantities like ratios of tensions of D-branes do not depend on this choice.

- The structure of the string field theory action (2.1) is very similar in spirit to the action of $p$-adic string theory [64, 65, 66]. Both are non-local, and in both cases the action expanded around the tachyon vacuum is perfectly non-singular and has no physical excitations. Yet in both cases the theory admits lump solutions which support open string excitations. The D-p-brane solutions are gaussian in the case of $p$-adic string theory, and also in the case of vacuum string field theory, although in this case the string field has many components corresponding to different oscillator excitations. It will be interesting to explore if there is any deeper significance to this similarity.

- Vacuum string field theory is much simpler than conventional cubic open string field theory. Explicit analytic solutions of equations of motion are possible, as we saw in this and the earlier papers. Also in this theory off-shell 'tachyon' amplitudes (and 
perhaps other amplitudes as well) around the tachyon vacuum can be computed exactly up to overall normalization. All this is commensurate with the fact that we have chosen to expand the action around a simpler background. In this context we would like to note that even the $p$-adic string action takes a simple form only when expended about the tachyon vacuum. Once we introduce shifted fields to expand the action around the D-brane background, the action looks much more complicated.

- Clearly the most pressing problem at this stage is understanding the ghost sector. This is needed not merely to complete the construction of the action, but also for understanding gauge transformations in this theory. This, in turn is needed for classifying inequivalent classical solutions and the spectrum of physical states around D-brane backgrounds. The knowledge of $\mathcal{Q}$ will also enable us to calculate quantum effects in this theory and determine whether the theory contains in its full spectrum, the elusive closed string states. On this issue, at the end of section 4.6 we speculated whether integration of dimension $(1,1)$ closed string primaries over the bulk of the sliver could yield a representation of closed string degrees of freedom in the open string state space.

In this paper we have seen that vacuum SFT incorporates nicely the most attractive features of boundary SFT - the automatic generation of correct tensions, and the description of solutions in terms of renormalization group ideas. These features arise in vacuum string field theory by taking into account the unusual geometrical definition of the sliver state. The two present shortcomings of boundary SFT - the tachyon vacuum being a singular endpoint of the configuration space, and the difficulty of defining the theory in the space of all backgrounds - are avoided in vacuum SFT. In a previous paper [31], we noted the remarkable simplicity of the algebraic approach to the construction of D-brane configurations in vacuum SFT. All in all, we are led to believe that vacuum string field theory may provide a surprisingly powerful and flexible approach to non-perturbative string theory.

Acknowledgements: We would like to thank J. David, D. Gaiotto, R. Gopakumar, F. Larsen, J. Minahan, S. Minwalla, N. Moeller, P. Mukhopadhyay, M. Schnabl, S. Shatashvili, S. Shenker, A. Strominger, W. Taylor, E. Verlinde and E. Witten for useful discussions. The figures were prepared by Marty Stock, and we are grateful to him for his careful work. The work of L.R. was supported in part by Princeton University "Dicke Fellowship" and by NSF grant 9802484. The work of A.S. was supported in part by NSF grant PHY99-07949. The work of B.Z. was supported in part by DOE contract \#DE-FC02-94ER40818. 


\section{References}

[1] A. Sen, "Descent relations among bosonic D-branes", Int. J. Mod. Phys. A14, 4061 (1999) hep-th/9902105.

[2] A. Sen, "Non-BPS states and branes in string theory", hep-th/9904207.

[3] A. Sen, "Universality of the tachyon potential", JHEP 9912, 027 (1999) hepth/9911116.

[4] E. Witten, "Noncommutative Geometry And String Field Theory", Nucl. Phys. B268, 253 (1986).

[5] V. A. Kostelecky and S. Samuel, "On A Nonperturbative Vacuum For The Open Bosonic String", Nucl. Phys. B 336, 263 (1990).

[6] A. Sen and B. Zwiebach, "Tachyon Condensation in String Field Theory", JHEP 0003, 002 (2000) hep-th/9912249.

[7] N. Moeller and W. Taylor, "Level truncation and the tachyon in open bosonic string field theory", Nucl. Phys. B583, 105 (2000) hep-th/0002237.

[8] J.A. Harvey and P. Kraus, "D-Branes as unstable lumps in bosonic open string field theory", JHEP 0004, 012 (2000) hep-th/0002117.

[9] R. de Mello Koch, A. Jevicki, M. Mihailescu and R. Tatar, "Lumps and p-branes in open string field theory", Phys. Lett. B482, 249 (2000) hep-th/0003031.

[10] N. Moeller, A. Sen and B. Zwiebach, "D-branes as tachyon lumps in string field theory", hep-th/0005036.

[11] L. Rastelli and B. Zwiebach, "Tachyon potentials, star products and universality", hep-th/0006240.

[12] A. Sen and B. Zwiebach, "Large marginal deformations in string field theory", JHEP 0010, 009 (2000) hep-th/0007153.

[13] W. Taylor, "Mass generation from tachyon condensation for vector fields on Dbranes", JHEP 0008, 038 (2000) hep-th/0008033.

[14] R. de Mello Koch and J.P. Rodrigues, "Lumps in level truncated open string field theory", hep-th/0008053. 
[15] N. Moeller, "Codimension two lump solutions in string field theory and tachyonic theories", hep-th/0008101.

[16] H. Hata and S. Shinohara, "BRST invariance of the non-perturbative vacuum in bosonic open string field theory". JHEP 0009, 035 (2000) hep-th/0009105.

[17] B. Zwiebach, "Trimming the Tachyon String Field with SU(1,1)", hep-th/0010190.

[18] M. Schnabl, "Constraints on the tachyon condensate from anomalous symmetries", hep-th/0011238.

[19] P. Mukhopadhyay and A. Sen, "Test of Siegel gauge for the lump solution", hepth/0101014.

[20] H. Hata and S. Teraguchi, "Test of the Absence of Kinetic Terms around the Tachyon Vacuum in Cubic String Field Theory", hep-th/0101162.

[21] I. Ellwood and W. Taylor, "Open string field theory without open strings", hepth/0103085.

[22] B. Feng, Y. He and N. Moeller, "Testing the uniqueness of the open bosonic string field theory vacuum", hep-th/0103103.

[23] K. Ohmori, "A review on tachyon condensation in open string field theories", hepth/0102085.

[24] I. Ellwood, B. Feng, Y. He and N. Moeller, "The identity string field and the tachyon vacuum", hep-th/0105024.

[25] K. Bardakci and M. B. Halpern, "Explicit spontaneous breakdown in a dual model", Phys. Rev. D10 (1974) 4230; K. Bardakci, "Spontaneous symmetry breaking in the standard dual string model", Nucl.Phys. B133 (1978) 297;

[26] B. Zwiebach, "A solvable toy model for tachyon condensation in string field theory", JHEP 0009, 028 (2000) hep-th/0008227;

J. A. Minahan and B. Zwiebach, "Field theory models for tachyon and gauge field string dynamics", JHEP 0009, 029 (2000) hep-th/0008231;

J. A. Minahan and B. Zwiebach, "Gauge fields and fermions in tachyon effective field theories", JHEP 0102, 034 (2001) hep-th/0011226. 
[27] P. Yi, "Membranes from five-branes and fundamental strings from Dp branes", Nucl. Phys. B 550, 214 (1999) hep-th/9901159];

A. Sen, "Supersymmetric world-volume action for non-BPS D-branes", JHEP 9910, 008 (1999) hep-th/9909062;

O. Bergman, K. Hori and P. Yi, "Confinement on the brane", Nucl. Phys. B 580, 289 (2000), hep-th/0002223;

G. Gibbons, K. Hori and P. Yi, "String fluid from unstable D-branes", Nucl. Phys. B 596, 136 (2001) hep-th/0009061;

A. Sen, "Fundamental strings in open string theory at the tachyonic vacuum", hepth/0010240.

[28] C. G. Callan, I. R. Klebanov, A. W. Ludwig and J. M. Maldacena, "Exact solution of a boundary conformal field theory", Nucl. Phys. B 422, 417 (1994) hep-th/9402113;

J. Polchinski and L. Thorlacius, "Free fermion representation of a boundary conformal field theory," Phys. Rev. D 50, 622 (1994) hep-th/9404008;

P. Fendley, H. Saleur and N. P. Warner, "Exact solution of a massless scalar field with a relevant boundary interaction," Nucl. Phys. B 430, 577 (1994) hep-th/9406125;

I. Affleck and A. W. Ludwig, "Universal noninteger 'ground state degeneracy' in critical quantum systems," Phys. Rev. Lett. 67, 161 (1991);

J. A. Harvey, D. Kutasov and E. J. Martinec, "On the relevance of tachyons," hepth/0003101;

S. Dasgupta and T. Dasgupta, "Renormalization group analysis of tachyon condensation," hep-th/0010247.

[29] L. Rastelli, A. Sen and B. Zwiebach, "String field theory around the tachyon vacuum", hep-th/0012251.

[30] L. Rastelli, A. Sen and B. Zwiebach, "Classical solutions in string field theory around the tachyon vacuum," hep-th/0102112.

[31] L. Rastelli, A. Sen and B. Zwiebach, "Half-strings, Projectors, and Multiple D-branes in Vacuum String Field Theory", hep-th/0105058.

[32] G. T. Horowitz, J. Morrow-Jones, S. P. Martin and R. P. Woodard, "New Exact Solutions For The Purely Cubic Bosonic String Field Theory", Phys. Rev. Lett. 60, 261 (1988).

[33] A. Kostelecky and R. Potting, "Analytical construction of a nonperturbative vacuum for the open bosonic string", hep-th/0008252. 
[34] C. G. Callan and I. R. Klebanov, "D-Brane Boundary State Dynamics," Nucl. Phys. B 465 (1996) 473 [hep-th/9511173].

[35] P. Di Vecchia, M. Frau, I. Pesando, S. Sciuto, A. Lerda and R. Russo, "Classical p-branes from boundary state," Nucl. Phys. B 507, 259 (1997) hep-th/9707068.

[36] S. Elitzur, E. Rabinovici and G. Sarkisian, "On least action D-branes," Nucl. Phys. B 541 (1999) 246 hep-th/9807161.

[37] J. A. Harvey, S. Kachru, G. Moore and E. Silverstein, "Tension is dimension," JHEP 0003, 001 (2000) hep-th/9909072.

[38] S. P. de Alwis, "Boundary string field theory, the boundary state formalism and D-brane tension," Phys. Lett. B 505, 215 (2001) hep-th/0101200.

[39] E. Witten, "On background independent open string field theory", Phys. Rev. D46, 5467 (1992) hep-th/9208027.

[40] E. Witten, "Some computations in background independent off-shell string theory", Phys. Rev. D47, 3405 (1993) hep-th/9210065.

[41] K. Li and E. Witten, "Role of short distance behavior in off-shell open string field theory", Phys. Rev. D48, 853 (1993) hep-th/9303067.

[42] S.L. Shatashvili, "Comments on the background independent open string theory", Phys. Lett. B311, 83 (1993) hep-th/9303143.

[43] S.L. Shatashvili, "On the problems with background independence in string theory", hep-th/9311177.

[44] J.A. Minahan and B. Zwiebach, "Field theory models for tachyon and gauge field string dynamics", JHEP 0009, 029 (2000) hep-th/0008231.

[45] A.A. Gerasimov and S.L. Shatashvili, "On exact tachyon potential in open string field theory", hep-th/0009103.

[46] D. Kutasov, M. Marino and G. Moore, "Some exact results on tachyon condensation in string field theory", hep-th/0009148.

[47] D. Ghoshal and A. Sen, "Normalisation of the background independent open string field theory action", hep-th/0009191. 
[48] A. A. Gerasimov and S. L. Shatashvili, "Stringy Higgs mechanism and the fate of open strings," JHEP 0101, 019 (2001) hep-th/0011009.

[49] D. J. Gross and W. Taylor, "Split string field theory I", hep-th/0105059.

[50] E. Witten, Private communications.

[51] K. Ranganathan, H. Sonoda and B. Zwiebach, "Connections on the state space over conformal field theories," Nucl. Phys. B 414, 405 (1994) hep-th/9304053.

[52] J. L. Cardy, "Boundary Conditions, Fusion Rules And The Verlinde Formula, Nucl. Phys. B 324, 581 (1989).

[53] E. Gava, K. S. Narain and M. H. Sarmadi, "On the bound states of p- and (p+2)branes," Nucl. Phys. B 504, 214 (1997) hep-th/9704006.

[54] A. Recknagel and V. Schomerus, "Boundary deformation theory and moduli spaces of D-branes," Nucl. Phys. B 545, 233 (1999) [hep-th/9811237.

[55] A. Sen and B. Zwiebach, "A proof of local background independence of classical closed string field theory," Nucl. Phys. B 414, 649 (1994) hep-th/9307088.

[56] C. Hong-Mo and T. Sheung Tsun, Phys. Rev. D 35, 2474 (1987);Phys. Rev. D 39, 555 (1989).

[57] J. Bordes, H. Chan, L. Nellen and S. T. Tsou, "Half string oscillator approach to string field theory", Nucl. Phys. B 351 (1991) 441.

[58] F. Anton, A. Abdurrahman and J. Bordes, "Half string oscillator approach to string field theory (ghost sector 1)," Nucl. Phys. B 397, 260 (1993)

[59] A. Abdurrahman, F. Anton and J. Bordes, "Half string oscillator approach to string field theory (ghost sector 2)," Nucl. Phys. B 411, 693 (1994).

[60] A. Abdurrahman and J. Bordes, "The relationship between the comma theory and Witten's string field theory. I," Phys. Rev. D 58, 086003 (1998).

[61] T. Kawano and K. Okuyama, "Open string fields as matrices," hep-th/0105129.

[62] B. Zwiebach, "Oriented open-closed string theory revisited," Annals Phys. 267, 193 (1998) hep-th/9705241;

B. Zwiebach, "Quantum open string theory with manifest closed string factorization," Phys. Lett. B 256, 22 (1991); 
B. Zwiebach, "Interpolating string field theories," Mod. Phys. Lett. A 7, 1079 (1992) [hep-th/9202015].

[63] A. Strominger, "Closed Strings In Open String Field Theory," Phys. Rev. Lett. 58 (1987) 629 .

[64] L. Brekke, P. G. Freund, M. Olson and E. Witten, "Nonarchimedean String Dynamics" Nucl. Phys. B302, 365 (1988).

[65] D. Ghoshal and A. Sen, "Tachyon condensation and brane descent relations in p-adic string theory", Nucl. Phys. B584, 300 (2000) [hep-th/0003278.

[66] J. Minahan, "Mode Interactions of the Tachyon Condensate in p-adic String Theory", hep-th/0102071. 